\title{
One-Dimensional Slow Invariant Manifolds for Fully Coupled Reaction and Micro-scale Diffusion*
}

\author{
J. D. Mengers ${ }^{\dagger}$ and J. M. Powers ${ }^{\ddagger}$
}

\begin{abstract}
The method of slow invariant manifolds (SIMs), applied previously to model the reduced kinetics of spatially homogeneous reactive systems, is extended to systems with diffusion. Through the use of a Galerkin projection, the governing partial differential equations are cast into a finite system of ordinary differential equations to be solved on an approximate inertial manifold. The SIM construction technique of identifying equilibria and connecting heteroclinic orbits is extended by identifying steady state solutions to the governing partial differential equations and connecting analogous orbits in the Galerkin-projected space. In parametric studies varying the domain length, the time scale spectrum is shifted, and various classes of nonlinear dynamics are identified. A critical length scale is identified below which the spatially homogeneous one-dimensional SIM models the long time dynamics of the system. At this critical length scale, a bifurcation in the slow dynamics of the system is identified; additional real nonsingular steady state solutions are found which lead to a diffusion-modified one-dimensional SIM. At these longer lengths, the spectral gap in the time scales indicates that an appropriate manifold for a reduction technique is higher than one-dimensional. This is shown for two examples: a simple chemical reaction mechanism, and the Zel'dovich reaction mechanism of $N O$ production. These examples are evaluated in the spatially homogeneous case (a one-term projection), a two-term projection capturing the coarsest effects of diffusion, and a high-order projection that is fully resolved.
\end{abstract}

Key words. slow invariant manifold, approximate inertial manifold, reaction-diffusion, chemical kinetics, model reduction, bifurcation

AMS subject classifications. 35B32, 35B42, 35K57, 57M50, 80A30

DOI. $10.1137 / 120877118$

1. Introduction. Numerical simulations of the partial differential equations (PDEs) that model multiscale continuum physics are prevalent across many fields of engineering. To obtain results with fidelity to the underlying continuum model, discrete simulations must generally resolve the entire range of scales present, both spatial and temporal; a large disparity in these scales is typically referred to as stiffness. The computational costs associated with these stiff simulations grow with the disparity of scales [1].

In recent decades, there have been efforts in model reduction techniques to decrease the computational costs of simulating reactive flows while maintaining as much consistency with

\footnotetext{
${ }^{*}$ Received by the editors May 14, 2012; accepted for publication (in revised form) by T. Kaper February 6, 2013; published electronically April 18, 2013. This work was partially supported by the National Science Foundation (NSF) under grant CBET-0650843.

http://www.siam.org/journals/siads/12-2/87711.html

${ }^{\dagger}$ Department of Aerospace and Mechanical Engineering, University of Notre Dame, Notre Dame, IN 46556 (jmengers@alumni.nd.edu). This author's research was supported by the University of Notre Dame's Department of Applied and Computational Mathematics and Statistics fellowship.

${ }^{\ddagger}$ Corresponding author. Department of Aerospace and Mechanical Engineering, University of Notre Dame, Notre Dame, IN 46556 (powers@nd.edu).
} 
the underlying physics as possible. The reviews of Griffiths [2] and Lu and Law [3] provide background information on many such methods which will not be considered here. Manifold methods are a class of reduction technique that attempt to reduce the computational cost by projecting dynamics onto lower-dimensional manifolds which are chosen to capture the long time dynamics of the system. The justification for manifold methods as a reduction technique is based on a phase space analysis in which families of nearby trajectories are predicted to collapse onto low-dimensional manifolds. Methods such as intrinsic low-dimensional manifold [4], computational singular perturbation (CSP) [5], and global quasi-linearization [6] identify a dynamical system's slow time scales to build a manifold; however, these methods do not create invariant manifolds. For a manifold to be invariant, the trajectory of an initial condition on the manifold must lie completely within the manifold for all later times. This is advantageous for a reduction technique since after the projection onto an invariant manifold, additional errors will not be incurred. The method of invariant manifolds [7] addresses this by using a thermodynamic projector to construct an invariant manifold. Other similar techniques such as minimal entropy production trajectory [8] and invariant constrained equilibrium edge [9] also construct invariant manifolds using equilibrium thermodynamic potentials. The slow invariant manifold (SIM) is another invariant manifold based on consideration of the local times scales of the system, which can be constructed using various techniques $[10,11,12$, $13,14,15,16,17,18,19]$. Al-Khateeb et al. [18] give a detailed discussion of these spatially homogeneous invariant manifold methods, which are applied to ordinary differential equations (ODEs).

For manifold methods to be incorporated into systems with transport, enhancements are required. These can be categorized into two types: (i) methods which use spatially homogeneous manifolds and then account for reaction-transport coupling, and (ii) methods that build manifolds based on the governing PDEs, which include transport. Methods of the first type include the Maas-Pope projection (MPP) [20, 21], the close-parallel assumption [22, 23], and a CSP extension to PDEs [24], which all use projector operators to account for diffusion while implementing spatially homogeneous manifold methods. The second type includes another CSP extension [25], approximate slow invariant manifold [26], and reaction-diffusion manifold [27], which each evaluate time scales so as to segregate the associated bases into fast and slow that are used to construct algebraic constraints defining slow manifolds for the governing PDE. Additional methods of the second type are a SIM extension [28, 29], where low-dimensional manifolds are built in the infinite-dimensional function space by using a predictor-corrector method modified from the MPP, and stretching-based reduction [30], which uses a normal stretching rate analysis of local linear dynamics to generate a local coordinate that segregates the slow stretching directions from the others.

We construct a SIM that is based on the governing PDEs of a reaction-diffusion system. We choose the SIM since it is a canonical manifold that isolates the slow dynamics. An intuitive expectation which follows from Robinson's squeezing property [31, Chap. 14] is that for trajectories to rapidly collapse onto the SIM, there must be a sufficient spectral gap in the time scales. This same separation of scales typically makes simulations costly. Using the SIM, however, allows us to leverage the time scale disparity; by identifying the slowest dynamics, the SIM accurately captures the long time behavior of the system. We will employ the construction technique pioneered by Davis and Skodje [13] for spatially homogeneous

Copyright (c) by SIAM. Unauthorized reproduction of this article is prohibited. 


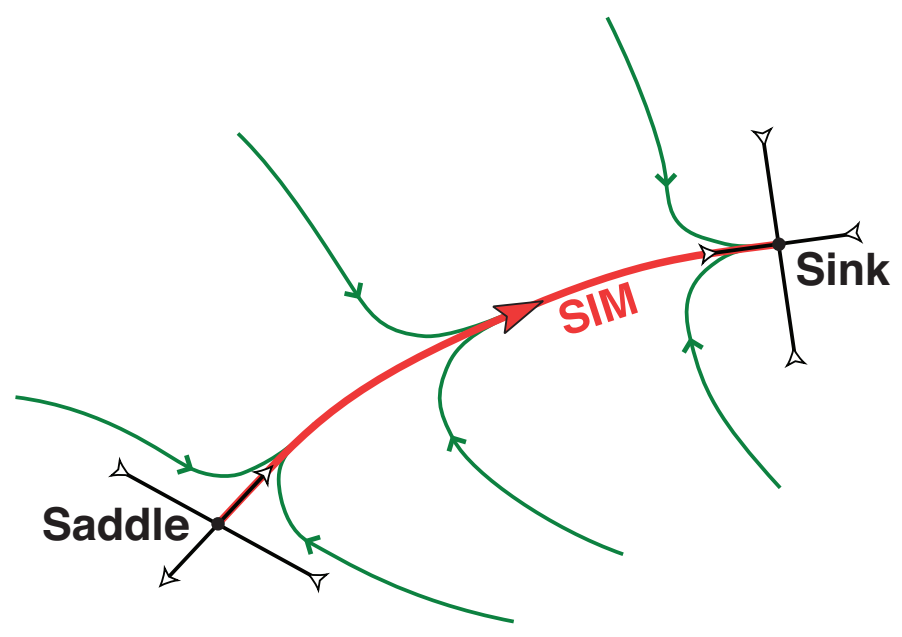

Figure 1. Sketch of one-dimensional SIM construction.

systems. In their method, branches of the one-dimensional SIM are identified as heteroclinic orbits connecting equilibria. One must first identify all equilibria of the system. A branch of the SIM is a heteroclinic orbit that connects a (nonphysical) saddle equilibrium that has one positive eigenvalue to the (physical) sink equilibrium. Trajectories are integrated from initial conditions that are perturbed from the nonphysical saddle along its only unstable eigenvector; any such trajectory that osculates with the eigenvector of the physical equilibrium whose eigenvalue corresponds to the slowest local time scale is a branch of the one-dimensional SIM. As sketched in Figure 1, the SIM connects the saddle to the sink, and trajectories originating away from the SIM are drawn toward it. We note that while this sketch often accurately represents a system's nonlinear dynamics, that there is no guarantee of global attraction of trajectories to the SIM.

We extend this SIM construction technique to reaction-diffusion systems by using the method of weighted residuals to reduce the governing PDEs into a system of ODEs; specifically, we employ the Galerkin method. We thus project solutions to the governing reaction-diffusion PDEs onto an approximate inertial manifold (AIM) [31, Chap. 14] and [32]. This is the same technique that Lorenz [33] used to simplify the Navier-Stokes equations. The resulting lowdimensional dynamical system has steady state solutions, or equilibria, that lend themselves to the same SIM construction techniques as spatially homogeneous systems. These equilibria in the Galerkin projection correspond to steady state solutions to the governing PDE. We then construct a one-dimensional SIM using heteroclinic orbits which approximates the time evolution of a function of the spatial variable from a nonphysical steady state to the physical equilibrium. Thus, our work is distinguished from previous reaction-diffusion reduction techniques, which were developed independent of inertial manifold theories [28, 30]. Finally, we note that a general and pertinent mathematical discussion of the nonlinear dynamics, bifurcation, and invariant manifolds of reactive-diffusive systems is given by Henry [34] with additional discussion given by Fusco and Hale [35].

In this method we construct a one-dimensional manifold to use in a reduction of the system. We find some cases, such as when the slowest reaction and slowest diffusion have similar time 
scales, where this one-dimensional approach might not suffice. Others [36, 37, 38, 39, 40] have also examined time scale relationships in reaction-diffusion systems in the context of reduction techniques: Kalachev et al. [36] compared the extreme cases where reaction was much faster than diffusion or vice versa for a simple reaction-diffusion system. Yannacopoulos et al. [37] use inertial manifolds to develop lumping methods for reduction. The novelty of the present work is in the examination of the SIM manifold method with diffusion across a range of length scales where the reaction and diffusion act at the same time scales.

Our method results in a concise analytical coupling of reaction and diffusion time scales. Other methods, such as operator splitting [41, 42, 43], consider reaction and diffusion independently. To apply operator splitting, one integrates reaction terms implicitly and separately integrates diffusion and advection terms explicitly. This contrasts with our approach, which is built upon an underlying physical coupling of reaction and diffusion processes. Our results will demonstrate a full coupling between reaction and diffusion length and time scales in a fashion consistent with Al-Khateeb, Powers, and Paolucci [44], as well as classical diffusion theory [45, p. 126]. This coupling of scales has implications on the necessary grid resolution in discrete simulations which implement spatially homogeneous manifold methods. The lower bound of wavelengths modeled is dependent on the spatial resolution; if a grid is too coarse, a spatially homogeneous manifold will fail to resolve wavelengths whose corresponding diffusion time scales are as slow as or slower than the slowest reaction time scales. Therefore, spatially homogeneous SIMs, which are constructed to model the slowest time scales, are inadequate for simulations with coarse grid resolution.

We model reaction-diffusion systems that exhibit an infinite spectrum of diffusion length scales and a finite set of reaction time scales. By treating the domain length as a variable parameter, we change the spectrum of length scales and, therefore, change the spectrum of diffusion time scales. This allows us to study various classes of behavior of reaction-diffusion systems. We will examine two cases: (i) a highly resolved 26 -term truncation that takes into account one spatially homogeneous mode and 25 modes with various spatial wavelengths (allowing us to examine the accuracy of lower truncations and to identify underlying consistencies between our method and the continuum model); and (ii) a two-term truncation that includes only the spatially homogeneous mode and the fundamental spatial mode. The advantage of this approach is that it is a low-dimensional, simple model that demonstrates the interesting dynamics of the interaction between reaction and diffusion; however, for large domain lengths, this truncation lacks spatial resolution. Despite the advantages of this method, it becomes computationally prohibitive for large reaction-diffusion systems and/or systems in multiple spatial dimensions.

This paper is structured into three sections. In section 2, mathematical background, we examine in detail the governing reaction-diffusion equations and a series of reductions to them; subsections 2.1 and 2.2 discuss the combustion modeling and assumptions, and subsection 2.3 discusses various reductions to our governing equations. In section 3 , methodology, we outline the extension of SIM to reaction-diffusion systems. In section 4, model problems, results are shown for two different chemical kinetic mechanisms, each coupled with a simple diffusion model. The first is a simple one-step kinetic mechanism, and the second is the two-step Zel'dovich reaction mechanism of $N O$ production. The second system's spatially homogeneous SIM was studied by Al-Khateeb et al. [18]. More generally stated, the present

Copyright (c) by SIAM. Unauthorized reproduction of this article is prohibited. 
study is largely an extension of [18] to include effects of diffusion.

2. Mathematical background. We are interested in a rational reduction of systems of PDEs of the form

$$
\frac{\partial \mathbf{z}}{\partial t}=\mathbf{f}(\mathbf{z})+\frac{\partial}{\partial x}\left(\mathbf{D} \cdot \frac{\partial \mathbf{z}}{\partial x}\right)
$$

where $\mathbf{z} \in \mathbb{R}^{R}$ are dependent variables, $\mathbf{f}$ are nonlinear functions that represent rates of change due to reaction, $\mathbf{D}$ is an $R \times R$ matrix of mass diffusivities, the temporal domain is $t \in[0, \infty)$, and there is only one spatial Cartesian dimension, $x \in[0, \ell]$. To enable a simpler analysis, we assume $\mathbf{D}$ is of the form $\mathbf{D}=D \mathbf{I}$, where $D$ is a scalar constant and $\mathbf{I}$ is the identity matrix. Then (2.1) can be cast as

$$
\frac{\partial \mathbf{z}}{\partial t}=\mathbf{f}(\mathbf{z})-\mathscr{L}(\mathbf{z})
$$

where $\mathscr{L}=-D \partial^{2} / \partial x^{2}$ is a self-adjoint, positive semidefinite, linear spatial differential operator that models diffusion. We choose to study homogeneous Neumann boundary conditions

$$
\left.\frac{\partial \mathbf{z}}{\partial x}\right|_{x=0}=\left.\frac{\partial \mathbf{z}}{\partial x}\right|_{x=\ell}=\mathbf{0}
$$

This choice will enable a direct comparison of reduction methods developed for spatially homogeneous systems to those that include diffusion. More general boundary conditions could be studied at the expense of introducing additional thin layers into the solution.

Following Finlayson [46], we approximate a solution to (2.2)-(2.3) by using separation of variables coupled with the method of weighted residuals. The dependent variables, $\mathbf{z}$, are approximated by a series of the product of time-dependent amplitudes, $\boldsymbol{\zeta}_{m}(t)$, and a set of spatial basis functions, $\phi_{m}(x)$ :

$$
\mathbf{z}(x, t) \approx \tilde{\mathbf{z}}_{M}(x, t)=\sum_{m=0}^{M} \boldsymbol{\zeta}_{m}(t) \phi_{m}(x),
$$

where $M$ is the number of terms in the approximation, and for each $m=0, \ldots, M, \boldsymbol{\zeta}_{m} \in \mathbb{R}^{R}$.

We can choose the basis functions to be the eigenfunctions of the diffusion operator,

$$
\mathscr{L}\left(\phi_{m}\right)=\mu_{m} \phi_{m} \quad \text { for } m=0, \ldots, M,
$$

where the eigenvalues, $\mu_{m}$, are guaranteed to be nonnegative and real, and the eigenfunctions orthogonal,

$$
\left\langle\phi_{m}(x), \phi_{n}(x)\right\rangle \begin{cases}=0 & \text { for } m \neq n, \\ \neq 0 & \text { for } m=n,\end{cases}
$$

in a Lebesgue space where the inner product is defined as $\left\langle\phi_{m}(x), \phi_{n}(x)\right\rangle=\int_{0}^{\ell} \phi_{m}(x) \phi_{n}(x) d x$. Note that the eigenfunctions could be normalized without loss of generality; however, we omit

Copyright $\odot$ by SIAM. Unauthorized reproduction of this article is prohibited. 
this process to allow for a direct comparison between solutions from the weighted residual approach and spatially homogeneous solutions to (2.1). The eigenfunctions are required to match the boundary conditions of (2.3), yielding

$$
\left.\frac{d \phi_{m}}{d x}\right|_{x=0}=\left.\frac{d \phi_{m}}{d x}\right|_{x=\ell}=0 .
$$

We also choose $\phi_{0}$ to be a spatially homogeneous function whose eigenvalue is zero, $\mu_{0}=0$. We then order the subsequent eigenfunctions by increasing eigenvalues (i.e., $\mu_{0} \leq \mu_{1} \leq \mu_{2} \ldots$.).

When the approximation from (2.4) is substituted into the form from (2.2), the result does not satisfy the equation exactly, but it will have a nonzero residual,

$$
\mathbf{r}(x, t)=\sum_{m=0}^{M} \frac{d \boldsymbol{\zeta}_{m}}{d t} \phi_{m}(x)-\mathbf{f}\left(\sum_{\hat{m}=0}^{M} \boldsymbol{\zeta}_{\hat{m}}(t) \phi_{\hat{m}}(x)\right)+\sum_{m=0}^{M} \mu_{m} \boldsymbol{\zeta}_{m}(t) \phi_{m}(x),
$$

when $M$ is finite. This residual is not the error, $\mathbf{e}(x, t)=\mathbf{z}(x, t)-\sum_{m=0}^{M} \boldsymbol{\zeta}_{m}(t) \phi_{m}(x)$; however, if the residual is zero, the error will be zero as well. To formulate evolution equations for the amplitudes, we take a series of $M$ spatially weighted averages of the residual and require each be zero,

$$
\left\langle\mathbf{r}(x, t), \psi_{m}(x)\right\rangle=0 \quad \text { for all } m=0, \ldots, M \text { and } t \in[0, \infty),
$$

where $\psi_{m}(x)$ is a set of $M$ spatial weighting functions. There are many viable choices for weighting functions. If we make the common choice of the basis functions $[31,32]$ as the weighting functions, $\psi_{m}(x)=\phi_{m}(x)$, our method is a Galerkin method. Substituting (2.8) (with dummy indices changed from $m$ to $n$ ) into (2.9) and distributing the inner product linear operator to each term in the residual yields

$$
\left\langle\sum_{n=0}^{M} \frac{d \boldsymbol{\zeta}_{n}}{d t} \phi_{n}(x), \phi_{m}(x)\right\rangle-\left\langle\mathbf{f}\left(\sum_{\hat{m}=0}^{M} \boldsymbol{\zeta}_{\hat{m}}(t) \phi_{\hat{m}}(x)\right), \phi_{m}(x)\right\rangle+\left\langle\sum_{n=0}^{M} \mu_{n} \boldsymbol{\zeta}_{n}(t) \phi_{n}(x), \phi_{m}(x)\right\rangle=0 .
$$

Further simplification removes spatially independent terms from the inner products and arranges the terms in the order that they were in (2.2),

$$
\sum_{n=0}^{M} \frac{d \boldsymbol{\zeta}_{n}}{d t}\left\langle\phi_{n}(x), \phi_{m}(x)\right\rangle=\left\langle\mathbf{f}\left(\sum_{\hat{m}=0}^{M} \boldsymbol{\zeta}_{\hat{m}}(t) \phi_{\hat{m}}(x)\right), \phi_{m}(x)\right\rangle-\sum_{n=0}^{M} \mu_{n} \boldsymbol{\zeta}_{n}(t)\left\langle\phi_{n}(x), \phi_{m}(x)\right\rangle .
$$

Because of the orthogonality of our basis functions, (2.11) can be reformulated to express the evolution of the amplitudes as

$$
\frac{d \boldsymbol{\zeta}_{m}}{d t}=\frac{\left\langle\mathbf{f}\left(\sum_{\hat{m}=0}^{M} \boldsymbol{\zeta}_{\hat{m}}(t) \phi_{\hat{m}}(x)\right), \phi_{m}(x)\right\rangle}{\left\langle\phi_{m}(x), \phi_{m}(x)\right\rangle}-\mu_{m} \boldsymbol{\zeta}_{m}(t) \quad \text { for } m=0, \ldots, M,
$$

which yields a system of $R(M+1)$ ODEs. We define the reactions' contribution to the amplitude evolution as

$$
\dot{\boldsymbol{\Omega}}_{m}\left(\boldsymbol{\zeta}_{\hat{m}}\right)=\frac{\left\langle\mathbf{f}\left(\sum_{\hat{m}=0}^{M} \boldsymbol{\zeta}_{\hat{m}}(t) \phi_{\hat{m}}(x)\right), \phi_{m}(x)\right\rangle}{\left\langle\phi_{m}(x), \phi_{m}(x)\right\rangle} .
$$

Copyright $\odot$ by SIAM. Unauthorized reproduction of this article is prohibited. 
To obtain the exact solution of (2.2), the residual must be driven to zero, which requires the limit of $M \rightarrow \infty$. In this sense, the PDE of (2.2) can be considered to be an infinite set of ODEs. While the exact solution to an infinite-dimensional system is intractable, approximations with finite $M$ project the trajectories of solutions to the infinite-dimensional system onto a finite-dimensional AIM [31]. The dynamics on this finite-dimensional approximation are governed by a system of ODEs,

$$
\frac{d \boldsymbol{\zeta}_{m}}{d t}=\dot{\boldsymbol{\Omega}}_{m}\left(\boldsymbol{\zeta}_{\hat{m}}\right)-\mu_{m} \boldsymbol{\zeta}_{m} \quad \text { for } m, \hat{m}=0, \ldots, M .
$$

Robinson [31, p. 387] shows that under certain conditions, inertial manifolds exponentially attract all of the trajectories of solutions to PDEs. Since the diffusion operator we are modeling is dissipative, and since we truncate the high frequency (and therefore fastest decaying) amplitudes in (2.1) and (2.2), we assume the AIM will also exponentially attract all trajectories of solutions to our PDEs. Our approximate solutions are obtained by integrating this finite-dimensional system of ODEs to obtain the amplitude evolution and then employing (2.4) to reconstruct an approximation of $\mathbf{z}(x, t)$. We find that these assumptions provide realistic approximations which compare favorably to well-resolved simulations. The evolution of amplitudes in (2.14) is forced by a nonlinear function, $\mathbf{F}_{m}\left(\boldsymbol{\zeta}_{\hat{m}}\right)=\dot{\boldsymbol{\Omega}}_{m}\left(\boldsymbol{\zeta}_{\hat{m}}\right)-\mu_{m} \boldsymbol{\zeta}_{m}(t)$, for $m, \hat{m}=0, \ldots, M$. The projection of the function $\mathbf{f}(\mathbf{z})$ onto the AIM, $\dot{\boldsymbol{\Omega}}_{m}\left(\boldsymbol{\zeta}_{\hat{m}}\right)$, represents the rate of change of each amplitude due to reaction, and the projection of $\mathscr{L}(\mathbf{z})$ onto the AIM, $\mu_{m} \boldsymbol{\zeta}_{m}(t)$, represents the contribution from diffusion.

In the case where the Galerkin projection is truncated at $M=0$, the spatially homogeneous case is recovered, and (2.14) reduces to

$$
\frac{d \zeta_{0}}{d t}=\dot{\Omega}_{0}\left(\boldsymbol{\zeta}_{0}\right)
$$

If our spatially homogeneous basis function is unity, $\phi_{0}=1$, then (2.15) is identical to the spatially homogeneous system in the original variables,

$$
\frac{d \mathbf{z}}{d t}=\mathbf{f}(\mathbf{z})
$$

Since many manifold methods, including the SIM, focus on the spatially homogeneous system, we consider this case for comparison. Since spatially homogeneous dynamics are present in a subspace of all truncations of this Galerkin projection, we can use the results from the higherorder truncations to identify deviations from the slow dynamics of the spatially homogeneous approximation. The next two sections (2.1 and 2.2) provide background for the physical equations which are modeled. Readers not interested in these details of physical chemistry are directed to section 2.3.

2.1. Governing equations. We now specialize (2.2)-(2.3) to consider a closed, isothermal, isochoric, reaction-diffusion system of ideal gases which are described by Dalton's law and detailed mass-action Arrhenius kinetics. Our system consists of $N$ species composed of $L$ elements interacting in $J$ reactions. We model one-dimensional spatial dynamics using Fick's law of diffusion with a single constant mass diffusivity and neglect advection. Our notation

Copyright (c) by SIAM. Unauthorized reproduction of this article is prohibited. 
closely follows that of Al-Khateeb et al. [18], where superscripts $(o),(*)$, and $(e)$ denote evaluation at reference pressure, initial state, and equilibrium, respectively, and quantities presented with an overbar $\left(^{-}\right)$denote evaluation on a per-mole basis.

The governing equations for our reaction-diffusion system are the species evolution equations,

$$
\rho \frac{\partial Y_{i}}{\partial t}=\overline{\mathrm{M}}_{i} \dot{\omega}_{i}-\frac{\partial j_{i}}{\partial x} \quad \text { for } i=1, \ldots, N
$$

where $Y_{i}, j_{i}, \dot{\omega}_{i}$, and $\overline{\mathrm{M}}_{i}$ are mass fraction, diffusive mass flux, molar production rate, and molecular mass of species $i$, respectively; $\rho$ is the density, which remains constant for this closed isochoric system. Note that $\dot{\omega}_{i}$ is standard notation for Arrhenius kinetics; the dot does not denote a time derivative.

We allow the initial conditions for each species to be arbitrary functions in space,

$$
Y_{i}(x, t=0)=Y_{i}^{*}(x) \text { for } i=1, \ldots, N,
$$

and we model homogeneous Neumann boundary conditions,

$$
\left.\frac{\partial Y_{i}}{\partial x}\right|_{x=0}=\left.\frac{\partial Y_{i}}{\partial x}\right|_{x=\ell}=0 \quad \text { for } i=1, \ldots, N
$$

We consider a general set of $J$ reactions with $N$ species, $\chi_{i}, i=1, \ldots, N$,

$$
\sum_{i=1}^{N} \nu_{i j}^{\prime} \chi_{i} \leftrightharpoons \sum_{i=1}^{N} \nu_{i j}^{\prime \prime} \chi_{i}
$$

where $j=1, \ldots, J$. The forward and reverse stoichiometric coefficients, $\nu_{i j}^{\prime}$ and $\nu_{i j}^{\prime \prime}$, are combined to a single net stoichiometric coefficient,

$$
\nu_{i j}=\nu_{i j}^{\prime \prime}-\nu_{i j}^{\prime},
$$

such that each reaction can be represented as

$$
\sum_{i=1}^{N} \chi_{i} \nu_{i j}=0 \text { for all } j=1, \ldots, J .
$$

Note that $\nu_{i j}$ has dimension $N \times J$ and rank $R$, where $R \leq N-L$. Stoichiometric constraints, which require elements to be conserved in each reaction, can be written as

$$
\sum_{i=1}^{N} \varphi_{l i} \nu_{i j}=0 \quad \text { for } l=1, \ldots, L \text { and } j=1, \ldots, J,
$$

where $\varphi_{l i}$ is the number of elements, $l$, in species $i$; this constrains $\nu_{i j}$ to be in the right null space of $\varphi_{l i}$. 
2.2. Constitutive equations. To complete the system, the following constitutive equations are specified. The mass flux is given by Fick's law of diffusion,

$$
j_{i}=-\rho D \frac{\partial Y_{i}}{\partial x} \quad \text { for } i=1, \ldots, N,
$$

where $D$ is the constant species mass diffusivity. The spatial gradient of mass flux is a selfadjoint, positive semidefinite, linear differential operator which is in the form of $\mathscr{L}$ from (2.14). As a result of (2.24), along with the homogeneous Neumann boundary conditions, there is no mass flux at either boundary. The pressure, $P$, is given by the ideal gas equation of state for a mixture that obeys Dalton's law:

$$
P=\rho \bar{\Re} T \sum_{i=1}^{N} \frac{Y_{i}}{\overline{\mathrm{M}}_{i}}
$$

where $\overline{\mathfrak{R}}=8.314 \times 10^{7} \mathrm{erg} /(\mathrm{mol} \mathrm{K})$ is the universal gas constant, and $T$ is the constant temperature. The reaction source terms, $\dot{\omega}_{i}$, are given by detailed mass-action Arrhenius kinetics:

$$
\begin{aligned}
\dot{\omega}_{i} & =\sum_{j=1}^{J} \nu_{i j} r_{j} \quad \text { for } i=1, \ldots, N, \\
r_{j} & =k_{j}\left(\prod_{i=1}^{N}\left(\frac{\rho Y_{i}}{\overline{\mathrm{M}}_{i}}\right)^{\nu_{i j}^{\prime}}-\frac{1}{K_{j}^{c}} \prod_{i=1}^{N}\left(\frac{\rho Y_{i}}{\overline{\mathrm{M}}_{i}}\right)^{\nu_{i j}^{\prime \prime}}\right) \text { for } j=1, \ldots, J, \\
k_{j} & =a_{j} T^{\beta_{j}} \exp \left(\frac{-\bar{E}_{j}}{\overline{\mathfrak{R} T}}\right) \quad \text { for } j=1, \ldots, J, \\
K_{j}^{c} & =\left(\frac{P^{o}}{\overline{\mathfrak{R} T}}\right)^{\sum_{i=1}^{N} \nu_{i j}} \exp \left(\frac{-\sum_{i=1}^{N} \bar{\mu}_{i}^{o} \nu_{i j}}{\overline{\mathfrak{R} T}}\right) \text { for } j=1, \ldots, J, \\
\bar{\mu}_{i}^{o} & =\overline{\mathrm{M}}_{i}\left(h_{i}-T s_{i}^{o}\right) \quad \text { for } i=1, \ldots, N,
\end{aligned}
$$

where $r_{j}, k_{j}$, and $K_{j}^{c}$ are the reaction rate, Arrhenius rate, and equilibrium constant of reaction $j$, respectively, and $\bar{\mu}_{i}^{o}$ is the chemical potential of species $i$ evaluated at the reference pressure, $P^{o}$. The Arrhenius rate depends on the collision frequency factor, $a_{j}$, the temperature-dependency exponent, $\beta_{j}$, and the activation energy, $\bar{E}_{j}$; the equilibrium constant depends on the reference pressure and chemical potentials; and the chemical potential depends on a specific enthalpy, $h_{i}$, and the specific entropy evaluated at the reference pressure, $s_{i}^{o}$. Equations (2.17)-(2.19) and (2.24)-(2.26) form a complete set.

2.3. Model reduction. In this section, we will show how the state variables in the governing and constitutive equations will reduce to a system of the form of (2.1) in the reduced species concentrations, $z_{i}$, which will be defined.

2.3.1. Generalized Shvab-Zel'dovich relations. First, we implement a reduction technique using the generalized Shvab-Zel'dovich relations similar to the reduction found in Lam 
and Bellan [47]. Similar to (2.23), we use a modified stoichiometric coefficient matrix, $\hat{\varphi}_{l i}$, that spans the left null space of matrix $\nu_{i j}$,

$$
\sum_{i=1}^{N} \hat{\varphi}_{l i} \nu_{i j}=0 \quad \text { for } l=1, \ldots, \hat{L} \text { and } j=1, \ldots, J,
$$

to replace $\hat{L}$ differential equations with algebraic constraints. We note that $\hat{L}=N-R$ because of the dimension and rank of $\nu_{i j}$. This reduction allows algebraic constraints (which enforce the stoichiometric constraints of the chemical system) to couple the evolution of $\hat{L}$ species to a reduced set of $R$ species. Note that $\hat{L} \geq L$ is the total number of linear constraints for the reaction mechanism, which includes all $L$ stoichiometric constraints, as well as any others. Operating on the molar production rates, (2.26a), with $\hat{\varphi}_{l i}$, and summing over all species, $i$, we get

$$
\sum_{i=1}^{N} \hat{\varphi}_{l i} \dot{\omega}_{i}=\sum_{i=1}^{N} \hat{\varphi}_{l i} \sum_{j=1}^{J} \nu_{i j} r_{j}=\sum_{j=1}^{J} r_{j} \sum_{i=1}^{N} \hat{\varphi}_{l i} \nu_{i j}=0 \quad \text { for } l=1, \ldots, \hat{L} .
$$

We apply the operator $\hat{\varphi}_{l i}$ to (2.17), use the diffusive mass flux from (2.24), and sum over all species to obtain

$$
\frac{\partial}{\partial t}\left(\sum_{i=1}^{N} \hat{\varphi}_{l i} \frac{Y_{i}}{\overline{\mathrm{M}}_{i}}\right)-D \frac{\partial^{2}}{\partial x^{2}}\left(\sum_{i=1}^{N} \hat{\varphi}_{l i} \frac{Y_{i}}{\overline{\mathrm{M}}_{i}}\right)=\sum_{i=1}^{N} \hat{\varphi}_{l i} \dot{\omega}_{i}=0 \quad \text { for } l=1, \ldots, \hat{L} .
$$

We now restrict our evaluation to systems whose initial conditions are spatially homogeneous in the values $\sum_{i=1}^{N} \hat{\varphi}_{l i} Y_{i} / \overline{\mathrm{M}}_{i}$, for $l=1, \ldots, \hat{L}$, which implies the initial element distribution is spatially homogeneous, as would typically be found in premixed combustion. Since the homogeneous Neumann boundary conditions yield no perturbations in the spatial distributions of these linear combinations of species, (2.29) can be integrated to yield

$$
\sum_{i=1}^{N} \hat{\varphi}_{l i} \frac{Y_{i}}{\overline{\mathrm{M}}_{i}}=\sum_{i=1}^{N} \hat{\varphi}_{l i} \frac{Y_{i}^{*}}{\overline{\mathrm{M}}_{i}} \quad \text { for } l=1, \ldots, \hat{L}
$$

therefore, these linear combinations of species will remain spatially homogeneous for all time. Note that individual species are not required to be spatially homogeneous; only the distribution of the linear constraints (i.e., elements) given in (2.30) has this requirement. The set of $\hat{L}$ algebraic relations implies that we need not solve for the dynamics of all $N$ species. Instead, we focus our attention on $R=N-\hat{L}$ reduced species and then use (2.30) to determine the dynamics of the remaining species.

2.3.2. Transformation to reduced variables. Following Ren et al. [9] and Al-Khateeb et al. [18], we now transform the mass fractions into specific mole concentrations, $\hat{z}_{i}$, where

$$
\hat{z}_{i} \equiv \frac{Y_{i}}{\overline{\mathrm{M}}_{i}} \quad \text { for } i=1, \ldots, N \text {. }
$$

Copyright $\odot$ by SIAM. Unauthorized reproduction of this article is prohibited. 
We can use the constraints in (2.30) to obtain a set of reduced variables. We choose the first $R$ linearly independent species as our reduced variables, $z_{n}, n=1, \ldots, R$, and then couple the values of $\hat{z}_{i}$ for $i=1, \ldots, N$ to those reduced variables by the linear relation

$$
\hat{z}_{i}(x, t)=\mathrm{z}_{i}+\sum_{n=1}^{R} \mathcal{D}_{i n} z_{n}(x, t) \quad \text { for } i=1, \ldots, N .
$$

Here $z_{n}$ are the $R$ reduced specific mole concentrations, $\mathbf{z}_{i}$ are the values of specific mole concentration when $z_{n}=0 \mathrm{~mol} / \mathrm{g}$ for all $n=1, \ldots, R$, and $\mathcal{D}_{i n}$ is a coefficient matrix of dimension $N \times R$ that couples the variations in $z_{n}$ to $\hat{z}_{i}$. We define $\mathcal{D}_{i n}$ to be an $N \times R$ full rank matrix that spans the column space of $\nu_{i j}, \sum_{i=1}^{N} \hat{\varphi}_{l i} \mathcal{D}_{i n}=0$ for $l=1, \ldots, \hat{L}$ and $n=1, \ldots, R$. This reduction is not unique. The following is a technique to obtain $\mathcal{D}_{i n}$ : reduce the transpose of $\nu_{i j}$ to a row-echelon form, truncate the final $J-R$ rows of zeros in this echelon form, and take the transpose of this truncation to form $\mathcal{D}_{i n}$. In this construction of $\mathcal{D}_{i n}$, the first $R$ rows form a submatrix which is an $R \times R$ identity matrix; this fact, along with the choice of $\mathrm{z}_{i}=0 \mathrm{~mol} / \mathrm{g}$ for $i=1, \ldots, R$, renders

$$
z_{i}=\hat{z}_{i} \quad \text { for } i=1, \ldots, R \text {. }
$$

This leaves the initial and boundary conditions in the reduced variables as

$$
z_{i}^{*}=\frac{Y_{i}^{*}}{\overline{\mathrm{M}}_{i}} \quad \text { for } i=1, \ldots, R
$$

and

$$
\left.\frac{\partial z_{i}}{\partial x}\right|_{x=0}=\left.\frac{\partial z_{i}}{\partial x}\right|_{x=\ell}=0 \quad \text { for } i=1, \ldots, R
$$

The remaining $\mathbf{z}_{i}, i=R+1, \ldots, N$, are chosen to satisfy the $\hat{L}$ algebraic constraints, (2.30), which are simplified to

$$
\sum_{i=1}^{N} \hat{\varphi}_{l i}\left(\mathrm{z}_{i}+\sum_{n=1}^{R} \mathcal{D}_{i n} z_{n}\right)=\sum_{i=1}^{N} \hat{\varphi}_{l i} z_{i}^{*} \quad \text { for } l=1, \ldots, \hat{L}
$$

and have the solution

$$
\mathrm{z}_{i}=z_{i}^{*}-\sum_{n=1}^{R} \mathcal{D}_{i n} z_{n}^{*} \quad \text { for } i=R+1, \ldots, N .
$$

Substituting specific mole concentrations, (2.31), along with the constitutive equation for mass flux, (2.24), into (2.17) yields $N$ evolution equations for $\hat{z}_{i}, i=1, \ldots, N$, where only the first $R$ equations are linearly independent. Therefore, we consider only the evolution equations for the reduced variables,

$$
\frac{\partial z_{i}}{\partial t}=\frac{\dot{\omega}_{i}\left(z_{n}\right)}{\rho}+D \frac{\partial^{2} z_{i}}{\partial x^{2}} \quad \text { for } i, n=1, \ldots, R,
$$

where we define a scaled chemical source term, $f_{i}\left(z_{n}\right)=\dot{\omega}_{i}\left(z_{n}\right) / \rho$, and diffusive flux term, $\mathscr{L}\left(z_{i}\right)=-D \partial^{2} z_{i} / \partial x^{2}$. Equation (2.38) is the final form considered and is in a form identical to $(2.2)$.

Copyright (c) by SIAM. Unauthorized reproduction of this article is prohibited. 
2.3.3. Galerkin reduction to ODEs. To analyze (2.38), we apply a Galerkin projection onto an AIM [31]. We accomplish this by assuming the reduced variables are approximated in the form of $(2.4)$,

$$
z_{i}(x, t) \approx \sum_{m=0}^{M} \zeta_{i, m}(t) \phi_{m}(x) \quad \text { for } i=1, \ldots, R,
$$

where $\phi_{m}(x)$ are chosen as scalar basis functions and $\zeta_{i, m}(t)$ are the corresponding timedependent amplitudes of species $i$. For a given domain length, we choose the truncation of our approximation by setting $M$ to a value above which the amplitude evolution is dominated by diffusion; diffusion causes the amplitudes, $\zeta_{i, m}$ for all $i$ and $m>M$, to decay rapidly before substantial reaction dynamics occur. We choose $\phi_{m}=\cos (m \pi x / \ell)$, which is a complete orthogonal basis in the limit as $M \rightarrow \infty$, and the corresponding eigenvalues are nonnegative and real, $\mu_{m}=D m^{2} \pi^{2} / \ell^{2}$. Our solution is decomposed into a Fourier cosine series whose $m=0$ amplitudes model the spatially homogeneous components and whose $m>0$ amplitudes model the spatial variations. By substituting (2.39) into (2.38) and taking the inner product with each basis function, $\phi_{n}(x)$, we obtain a finite system of ODEs for the evolution of the amplitudes in the form of (2.12). Since our molar production rates are low-order polynomials and our basis functions are cosines, the integration of the $\dot{\Omega}_{m}\left(\zeta_{\hat{m}}\right)$ terms in (2.13) can be performed analytically using trigonometric identities, as shown in Appendix A. We can therefore focus on the evaluation of our finite-dimensional system in the form of (2.14),

$$
\frac{d \zeta_{i, m}}{d t}=\dot{\Omega}_{i, m}\left(\zeta_{j, n}\right)-\frac{D m^{2} \pi^{2}}{\ell^{2}} \zeta_{i, m} \quad \text { for } i, j=1, \ldots, R \text { and } m, n=0, \ldots, M .
$$

The initial conditions of the reduced species amplitudes are given by

$$
\zeta_{i, m}(0)=\frac{\left\langle\phi_{m}, z_{i}^{*}\right\rangle}{\left\langle\phi_{m}, \phi_{m}\right\rangle} \quad \text { for } i=1, \ldots, R \text { and } m=0, \ldots, M \text {. }
$$

For very small $\ell$, a truncation at $M=0$ is appropriate, in which case (2.40) and (2.41) reduce to a spatially homogeneous system in the form of (2.16),

$$
\begin{aligned}
\frac{d z_{i}}{d t} & =f_{i}\left(z_{n}\right) \quad \text { for } i, n=1, \ldots, R, \\
z_{i}(0) & =z_{i}^{*} \quad \text { for } i=1, \ldots, R .
\end{aligned}
$$

For this to be the case, $\ell$ must be sufficiently small such that the diffusion term, $D m^{2} \pi^{2} / \ell^{2}$, will dominate the evolution equation for $m \geq 1$. At this small length scale, diffusion alone will cause all spatial inhomogeneities to equilibrate quickly, and the remaining reaction dynamics can be accurately modeled as the spatially homogeneous system given in (2.42)-(2.43).

3. Methodology. In this section we describe our extension of SIM construction from spatially homogeneous systems to reaction-diffusion systems and describe some of the ramifications of our approach. The approach we take to SIM construction is an extension of the spatially homogeneous technique given by Davis and Skodje [13] and Al-Khateeb et al. [18]. This technique constructs individual branches of the one-dimensional SIM by integrating a

Copyright (c) by SIAM. Unauthorized reproduction of this article is prohibited. 
heteroclinic orbit from a nonphysical saddle equilibrium, along its only unstable eigenvector, to the physical equilibrium sink, which the SIM approaches along the equilibrium's slowest eigenvector. We label the nonphysical saddle equilibrium from which a SIM branch emanates the SIM branch initial condition (SIM-BIC). There is no guarantee that SIM-BICs exist for every chemical mechanism. The evaluation of the entire dynamical system, including the equilibria with negative concentrations and other nonphysical mathematical artifacts, provides useful insights into the physical dynamics, as shown by Davis and Skodje [13] and Al-Khateeb et al. [18].

This technique is extended to the infinite-dimensional PDEs governing a reaction-diffusion equation by use of the Galerkin projection onto an AIM, as described in section 2.3.3. This AIM is an $R(M+1)$-dimensional system of ODEs, whose equilibria are amenable to a SIM construction technique similar to that used for the spatially homogeneous system of ODEs. Here we make a distinction between our terminology of equilibrium and chemical equilibrium. What we call an equilibrium is a fixed point in our finite-dimensional projection of an infinitedimensional dynamical system which is the steady state solution to (2.2),

$$
\mathbf{f}\left(\mathbf{z}^{e}\right)-\mathscr{L}\left(\mathbf{z}^{e}\right)=\mathbf{0}
$$

with boundary conditions that match (2.3),

$$
\left.\frac{\partial \mathbf{z}}{\partial x}\right|_{x=0}=\left.\frac{\partial \mathbf{z}}{\partial x}\right|_{x=\ell}=\mathbf{0} .
$$

This solution, or equilibrium manifold, is a spatial function, $\mathbf{z}^{e}(x)$, which can be approximated by the steady state solution of the Galerkin projection in (2.14),

$$
\mathbf{F}_{m}\left(\boldsymbol{\zeta}_{\hat{m}}^{e}\right)=\dot{\boldsymbol{\Omega}}_{m}\left(\boldsymbol{\zeta}_{\hat{m}}^{e}\right)-\mu_{m} \boldsymbol{\zeta}_{m}^{e}=\mathbf{0} \text { for } m, \hat{m}=0, \ldots, M \text {. }
$$

The solution to (3.3) is a fixed point in the finite-dimensional phase space which can be reconstructed into the approximation $\tilde{\mathbf{z}}_{M}^{e}(x)$ using (2.4). In the $M=0$ truncation, the spatially homogeneous reaction-only equilibria are recovered,

$$
\mathbf{f}\left(\mathbf{z}^{e: S H}\right)=\mathbf{0} .
$$

The concentrations of one of these spatially homogeneous equilibria are equal to their chemical equilibrium values.

The spatially homogeneous equilibria, $\mathbf{z}^{\mathrm{e}: S H}$, which satisfy the steady state $M=0$ Galerkin projection, (3.4), will have no contribution from the diffusion operator $\mathscr{L}\left(\mathbf{z}^{e: S H}\right)=\mathbf{0}$ and will also satisfy the full steady state equation, (3.1). The spatially homogeneous equilibria are also exact steady state solutions to any Galerkin projection, where $\boldsymbol{\zeta}_{0}=\mathbf{z}^{e: S H}$ and $\boldsymbol{\zeta}_{m}=\mathbf{0}$ for $m>0$. Furthermore, all spatially homogeneous dynamics of the $M>0$ Galerkin projections occur in a subspace of these larger AIMs; when $\zeta_{i, m}=0$ for all $i$ and for $m>0$, all dynamics occur on the $M=0$ AIM, which we call the spatially homogeneous subspace.

For mathematical completeness, we seek additional, spatially inhomogeneous equilibria to the steady state Galerkin projection, (3.3). Since these solutions require a nontrivial balance between the irreversible processes of reaction and diffusion, and since we choose to model

Copyright (c) by SIAM. Unauthorized reproduction of this article is prohibited. 
zero-flux boundary conditions, (2.3), the only spatially inhomogeneous equilibria allowed are nonphysical. This is because the entropy generated in balancing reaction and diffusion has no means of transferring out of the domain. A different choice of boundary conditions would remove this restriction.

Since the reaction-diffusion AIM projection has a higher dimension than the spatially homogeneous system, its Jacobian has additional eigenvalues. We analytically examine the additional eigenvalues at an arbitrary point in the spatially homogeneous subspace, focusing first on the spatially homogeneous equilibria. From local linear analysis of the spatially homogeneous system, we find a reaction-only Jacobian matrix defined as

$$
J_{i j}=\frac{\partial f_{i}}{\partial z_{j}} \quad \text { for } i, j=1, \ldots, R .
$$

This Jacobian has $R$ eigenvalues, $\lambda_{0, i}, i=1, \ldots, R$, which we sort from largest to smallest by their real parts $\left(\operatorname{Re}\left(\lambda_{0, i}\right) \geq \operatorname{Re}\left(\lambda_{0, i+1}\right)\right.$ for $\left.i=1, \ldots, R-1\right)$. By defining the local chemical time scales as the reciprocal of the eigenvalues,

$$
\tau_{i, 0}=\frac{1}{\left|\lambda_{i, 0}\right|} \quad \text { for } i=1, \ldots, R,
$$

we see that when all eigenvalues have negative real parts (as they do in the neighborhood of the physical equilibrium), this ordering becomes slowest (at $i=1$ ) to fastest (at $i=R$ ). Local linear analysis of any point in the spatially homogeneous subspace in $M>0$ truncations of the Galerkin projection, (2.40), yields a Jacobian matrix that is of block diagonal form,

$$
\mathbf{J}_{\mathrm{AIM}}=\left[\begin{array}{cccccc}
\mathbf{J} & \mathbf{0} & \ldots & \mathbf{0} & \ldots & \mathbf{0} \\
\mathbf{0} & \mathbf{J}-\frac{\pi^{2} D}{\ell^{2}} \mathbf{I} & \ldots & \mathbf{0} & \ldots & \mathbf{0} \\
\vdots & \vdots & \ddots & \vdots & & \vdots \\
\mathbf{0} & \mathbf{0} & \ldots & \mathbf{J}-\frac{m^{2} \pi^{2} D}{\ell^{2}} \mathbf{I} & \ldots & \mathbf{0} \\
\vdots & \vdots & & \vdots & \ddots & \vdots \\
\mathbf{0} & \mathbf{0} & \ldots & \mathbf{0} & \ldots & \mathbf{J}-\frac{M^{2} \pi^{2} D}{\ell^{2}} \mathbf{I}
\end{array}\right]
$$

as seen from the derivation in Appendix B, where $\mathbf{J}$ is the reaction-only Jacobian matrix evaluated at the spatially homogeneous concentrations, and $\mathbf{I}$ and $\mathbf{0}$ are identity and zero $R \times R$ square matrices, respectively.

The eigenvalues of this block diagonal Jacobian matrix are correlated to the $R$ reactiononly eigenvalues of $\mathbf{J}$ and are given by

$$
\lambda_{i, m}=\lambda_{i, 0}-\frac{m^{2} \pi^{2} D}{\ell^{2}} \quad \text { for } i=1, \ldots, R \text { and } m=0, \ldots, M .
$$

We label these the diffusion-modified eigenvalues for $m \geq 1$. Using the same definition of local time scales as in the spatially homogeneous case, we find that

$$
\tau_{i, m}=\left|\lambda_{i, 0}-\frac{m^{2} \pi^{2} D}{\ell^{2}}\right|^{-1} \quad \text { for } i=1, \ldots, R \text { and } m=0, \ldots, M,
$$

Copyright (c) by SIAM. Unauthorized reproduction of this article is prohibited. 
where we see that these diffusion-modified time scales have a contribution from reaction, $\lambda_{i, 0}$, and a contribution from diffusion, $\mu_{m}^{2} D$; therefore, we define a diffusion time scale for each spatially inhomogeneous Fourier mode:

$$
\tau_{D, m}=\frac{\ell^{2}}{m^{2} \pi^{2} D} \quad \text { for } m=1, \ldots, M
$$

Since the diffusion contribution to the modified eigenvalues is always negative, the addition of diffusion provides a stabilizing effect (the eigenvalues become more negative). For equilibria with positive chemical eigenvalues, $\lambda_{i, 0}>0$, (3.8) identifies critical length scales where the diffusion-modified eigenvalue will be zero, $\ell_{c}=m \pi \sqrt{D \tau_{i, 0}}$; this result is similar to that of Al-Khateeb, Powers, and Paolucci [44].

We note that our boundary conditions and basis functions allow a symmetry in the dynamics in the Galerkin projection of our system. The initial conditions of $z_{i}^{*}(x)$ that satisfy the homogeneous Neumann boundary conditions will admit the evolution of all $z_{i}(x, t)$ for all $i=1, \ldots, R$. A symmetric initial condition, $z_{i}^{*}(\ell-x)$, will also satisfy the boundary conditions and will have a symmetric evolution of $z_{i}(\ell-x, t)$ that satisfies the governing PDE. When projected into the Fourier domain, the amplitudes of $z_{i}(x, t)$ are $\zeta_{i, m}(t)$, while the amplitudes of $z_{i}(\ell-x, t)$ are $(-1)^{m} \zeta_{i, m}(t)$. This symmetry means that phase space will have a reflective symmetry about the spatially homogeneous subspace, $\zeta_{i, m}(t)=0$ for all $i=1, \ldots, R$ and $m=1, \ldots, M$. We restrict our analysis to the half of phase space where $\zeta_{1,1}(t) \geq 0$ since the dynamics of the other half will be symmetric. We clarify that this discussion is describing two solutions which exhibit a reflective symmetry about $x=\ell / 2$, not whether solutions themselves are symmetric or asymmetric.

\section{Model problems.}

4.1. Simple chemical mechanism. In this section we will examine a simple problem with $R=1$ in the form of (2.2). We then identify steady state solutions and their linear stability, find a critical length scale where a supercritical pitchfork bifurcation occurs, and show spectral convergence of the solutions as more Galerkin modes are included. Then, we identify SIMBICs as the stable branches of the pitchfork bifurcation and compare the phase portraits of the spatially homogeneous system and $M=1$ Galerkin projection.

The governing equation we model is

$$
\frac{\partial z}{\partial t}=-K\left(z-C_{1}\right)\left(z-C_{2}\right)+D \frac{\partial^{2} z}{\partial x^{2}}
$$

where $K>0$ and $C_{1} \leq 0<C_{2}$, and whose boundary conditions are consistent with (2.3),

$$
\left.\frac{\partial z}{\partial x}\right|_{x=0}=\left.\frac{\partial z}{\partial x}\right|_{x=\ell}=0
$$

We take $z^{e}(x)$ to be a steady solution to (4.1)-(4.2) and evaluate its linear stability. We assume the initial conditions are perturbed from the steady state solution, $z(x, 0)=z^{e}(x)+\epsilon A(x, 0)$, where $A(x, 0)$ is $\mathcal{O}(1)$, and $0<\epsilon \ll 1$, and evaluate the subsequent evolution of

$$
z(x, t)=z^{e}(x)+\epsilon A(x, t) .
$$

Copyright (c) by SIAM. Unauthorized reproduction of this article is prohibited. 
This is equivalent to linearizing this equation around the steady state, $z^{e}(x)$. When we substitute the solution from (4.3) into (4.1), collect the terms that are $\mathcal{O}(\epsilon)$, and assume a separation of variables, $A(x, t)=G(x) H(t)$, we obtain two linear ODEs,

$$
\begin{aligned}
\frac{d H}{d t} & =\gamma H(t), \\
\frac{d^{2} G}{d x^{2}} & =\left(\frac{\gamma+K\left(2 z^{e}(x)-C_{1}-C_{2}\right)}{D}\right) G(x),
\end{aligned}
$$

where $\gamma$ is a constant. This result is identical to the Galerkin method for the PDE linearized about $z^{e}(x)$. The solution to (4.4) is an exponential, $H(t)=H(0) \exp (\gamma t)$, where the sign of $\gamma$ indicates the stability of the solution. The solution to (4.5) for a general steady state equilibrium, $z^{e}(x)$, typically requires numerical evaluation; however, if we evaluate spatially homogeneous equilibria, $z^{e}(x)=z^{e: S H}$, the solutions to (4.5) that match the boundary conditions are of the form

$$
G(x)=\cos \left(\sqrt{\frac{-\gamma-K\left(2 z^{e: S H}-C_{1}-C_{2}\right)}{D}} x\right),
$$

where the coefficient of $x$ in the argument of the cosine function must be an integer multiple of $\pi / \ell$. Therefore, we can solve for the stability parameter, $\gamma$, which yields the spectrum of values,

$$
\gamma=-K\left(2 z^{e: S H}-C_{1}-C_{2}\right)-\frac{m^{2} \pi^{2} D}{\ell^{2}}
$$

for any integer value of $m \geq 0$. Note that $\gamma$ is composed of two terms: $\lambda_{0}=-K\left(2 z^{e: S H}-\right.$ $C_{1}-C_{2}$ ), which is the eigenvalue of the reaction-only system, and $-\mu_{m}^{2} D=-m^{2} \pi^{2} D / \ell^{2}$, which is the diffusion-modification term. Therefore, the linear stability analysis for a spatially homogeneous equilibrium is consistent with the Jacobian eigenvalue analysis in section 3 . We also find that for a spatially inhomogeneous solution, the eigenvalues of the Jacobian of the Galerkin projection are a good approximation of the stability parameter, $\gamma$.

By inspection, we find two spatially homogeneous steady state solutions to (4.1)-(4.2) with finite concentrations, $z^{e: S H}=C_{1}$ and $z^{e: S H}=C_{2}$. There are other steady solutions with finite concentrations which are spatially inhomogeneous; their composition and stability are dependent on the reaction parameters and the domain length, which will be illustrated in an upcoming example. The reaction-only eigenvalue of the spatially homogeneous equilibrium, $z^{e: S H}=C_{2}$, is negative, $\lambda_{0}=-K\left(C_{2}-C_{1}\right)<0$, and the diffusion-modification contribution is nonpositive; therefore, all values of $\gamma$ are negative, and $z^{e: S H}=C_{2}$ is stable. The equilibrium $z^{e: S H}=C_{1}$ has a positive reaction-only eigenvalue, $\lambda_{0}=-K\left(C_{1}-C_{2}\right)>0$. Since the $m=0$ mode has no diffusion contribution, $\gamma$ for this mode will also be positive, and $z^{e: S H}=C_{1}$ will be unstable for at least one mode; however, there is an infinite spectrum of values for $\gamma$, so for any finite domain length, there will be modes where the negative diffusion contribution has stabilized the positive reaction contribution, resulting in a stable mode. The number of unstable modes depends on the domain length $\ell$.

Copyright $@$ by SIAM. Unauthorized reproduction of this article is prohibited. 
We now examine a simple reaction mechanism,

$$
B+B \leftrightharpoons A+A
$$

with $N=2$ species, $J=1$ reaction, and $\hat{L}=1$ constraint, which can be modeled by a system in the form of (4.1). By choosing $i=\{1,2\}$ to correspond to the species $\{A, B\}$, respectively, the rate equation for this reaction is

$$
r=k\left(\left(\frac{\rho Y_{2}}{\overline{\mathrm{M}}}\right)^{2}-\frac{1}{K^{c}}\left(\frac{\rho Y_{1}}{\overline{\mathrm{M}}}\right)^{2}\right) .
$$

The mole fractions must sum to unity, $Y_{1}+Y_{2}=1$, enabling the reduction shown in section 2.3. Since $R=N-\hat{L}=1$, we choose to model the evolution of species $A$ as the reduced variable $z=Y_{1} / \bar{M}$ and algebraically solve for the evolution of $B$ with the mole fraction constraint. This yields the evolution equation in the form of (2.38),

$$
\frac{\partial z}{\partial t}=2 \rho k\left(\left(\frac{1}{\bar{M}}-z\right)^{2}-\frac{z^{2}}{K^{c}}\right)+D \frac{\partial^{2} z}{\partial x^{2}} .
$$

We choose parameters to induce scales that are loosely correlated to those found in reactive gases at atmospheric pressure, $k=2.5 \times 10^{8} \mathrm{~cm}^{3} / \mathrm{mol} / \mathrm{s}, \overline{\mathrm{M}}=1 \mathrm{~g} / \mathrm{mol}, K^{c}=0.25, \rho=$ $1 \times 10^{-3} \mathrm{~g} / \mathrm{cm}^{3}$, and $D=1 \mathrm{~cm}^{2} / \mathrm{s}$. The resulting evolution equation is in the form of (4.1) with parameters $K=2 k \rho\left(1-1 / K^{c}\right)=1.5 \times 10^{6} \mathrm{~g} / \mathrm{mol} / \mathrm{s}, C_{1}=\sqrt{K^{c}} /\left(\left(\sqrt{K^{c}}-1\right) \overline{\mathrm{M}}\right)=-1 \mathrm{~mol} / \mathrm{g}$, and $C_{2}=\sqrt{K^{c}} /\left(\left(\sqrt{K^{c}}+1\right) \overline{\mathrm{M}}\right)=1 / 3 \mathrm{~mol} / \mathrm{g}$ :

$$
\frac{\partial z}{\partial t}=-1.5 \times 10^{6}(z+1)\left(z-\frac{1}{3}\right)+\frac{\partial^{2} z}{\partial x^{2}}
$$

This system has two finite spatially homogeneous equilibria,

$$
z^{e: S H}=\left\{-1, \frac{1}{3}\right\} \mathrm{mol} / \mathrm{g}
$$

which we label $R_{1}$ and $R_{2}$, respectively. The reaction-only eigenvalues of the linearized system about these equilibria are $\lambda_{0}=2 \times 10^{6} \mathrm{~s}^{-1}$ at $R_{1}$ and $\lambda_{0}=-2 \times 10^{6} \mathrm{~s}^{-1}$ at $R_{2}$. We know that $R_{2}$ is linearly stable since all of the values of $\gamma$ from its linear stability analysis are negative, independent of domain length. This result for the stability, along with the fact that both species concentrations are positive, indicates that $R_{2}$ is the physical equilibrium. Evaluating $R_{1}$, we find that the $m=0$ mode is unstable, but the sign of $\gamma$ for larger values of $m$ depends on the domain length.

In addition to evaluating the stability of the spatially inhomogeneous modes at $R_{1}$, we now find any additional spatially inhomogeneous steady solutions and evaluate their stability. The Galerkin projection admits an infinite number of steady solutions in the $M \rightarrow \infty$ limit. Most of these equilibria are complex and/or singular; we are interested only in the real nonsingular steady state solutions. By identifying all the roots with an algebraic analysis for a loworder system ( $M=3$ Galerkin projection) and resolving these roots to a higher-order system

Copyright (C) by SIAM. Unauthorized reproduction of this article is prohibited. 
(a)

$\ell=22.5 \mu m$

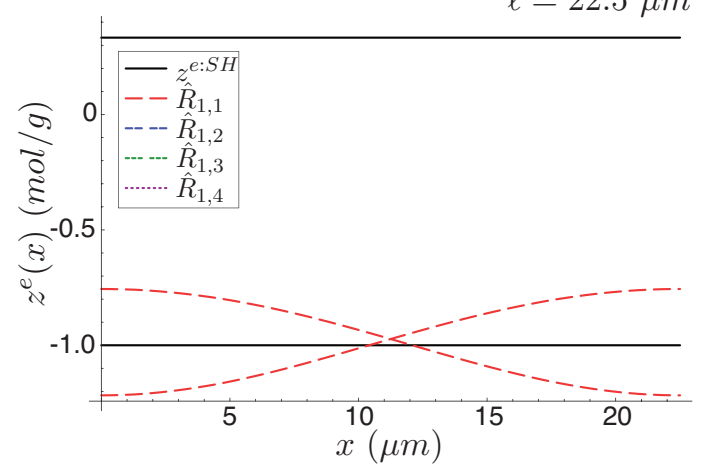

(b)

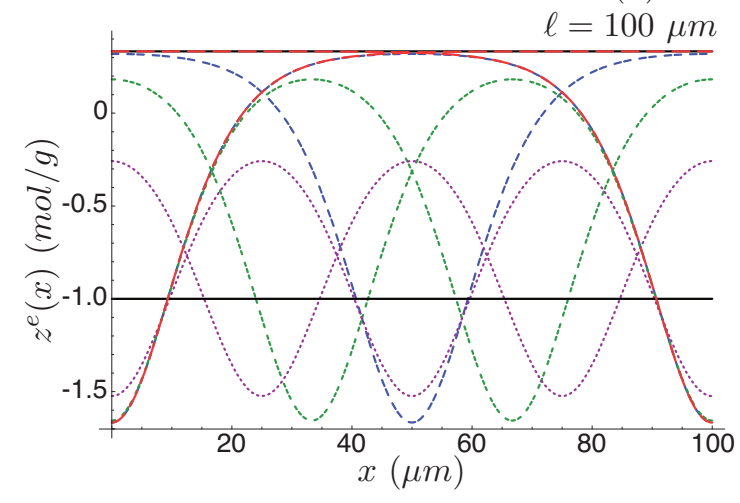

Figure 2. Steady state solutions for the $B+B \leftrightharpoons A+A$ mechanism.

$(M=25)$ using Newton's method, we find that the number of real nonsingular solutions increase as $\ell$ increases. We present the results for three different domain lengths: $\ell=5 \mu \mathrm{m}$, $22.5 \mu \mathrm{m}$, and $100 \mu \mathrm{m}$.

For $\ell=5 \mu \mathrm{m}$, the only steady state solutions we identify are the spatially homogeneous equilibria, $R_{1}$ and $R_{2}$; we find $R_{1}$ to have only one positive value of $\gamma$ for $m=0$, and it is therefore unstable only in the spatially homogeneous mode. For $\ell=22.5 \mu \mathrm{m}$, we find that $R_{1}$ is unstable in two modes $(m=0$ and $m=1)$; also, we identify two real nonsingular spatially inhomogeneous steady solutions, which each have only one positive value of $\gamma$. For $\ell=100 \mu m, R_{1}$ has five unstable modes for $m=0$ through $m=4$. At this length we identify eight real nonsingular spatially inhomogeneous steady solutions, all of which are unstable in some modes; two of these solutions have one positive value of $\gamma$, two have two, two have three, and two have four. The spatial reconstructions of the steady solutions in the $M=25$ Galerkin approximation are shown in Figure 2 for $\ell=22.5 \mu \mathrm{m}$ and for $\ell=100 \mu \mathrm{m}$, where the value $m$ in $\hat{R}_{1, m}$ indicates the number of unstable modes in the spatially inhomogeneous solutions. Some of the solutions for $\ell=100 \mu \mathrm{m}$ with low values of $m$ are quite similar and are therefore hard to discern in Figure 2; the spatially homogeneous $R_{2}$ at $z=1 / 3 \mathrm{~mol} / \mathrm{g}$ (black) and one $\hat{R}_{1,2}$ (blue dash-dashed) are both nearly obscured by half of each $\hat{R}_{1,1}$ solution (red dashed), which cross at $x=50 \mu \mathrm{m}$.

We see from these examples that for larger values of $\ell$, more of the modes in the neighborhood of $R_{1}$ become unstable. These transitions from stability to instability occur at integer multiples of a critical domain length,

$$
\ell_{c}=\pi \sqrt{\frac{D}{\lambda_{0}}}=\frac{\pi}{\sqrt{2 \times 10^{6}}}=22.214 \mu m
$$

where the $m=1$ diffusion-modification time scale is equal to the unstable reaction time scale. The critical length scale is coupled to a chemical time scale by diffusion: $\ell_{c}=\pi \sqrt{D \tau_{0}}$.

Therefore, when $\ell<\ell_{c}, R_{1}$ will have one positive eigenvalue and will in fact be a SIM$\mathrm{BIC}$ for the system; however, for domain lengths longer than $\ell_{c}, R_{1}$ will have more than one positive eigenvalue and will no longer qualify as a candidate equilibrium for the SIM-BIC. We

Copyright $\odot$ by SIAM. Unauthorized reproduction of this article is prohibited. 


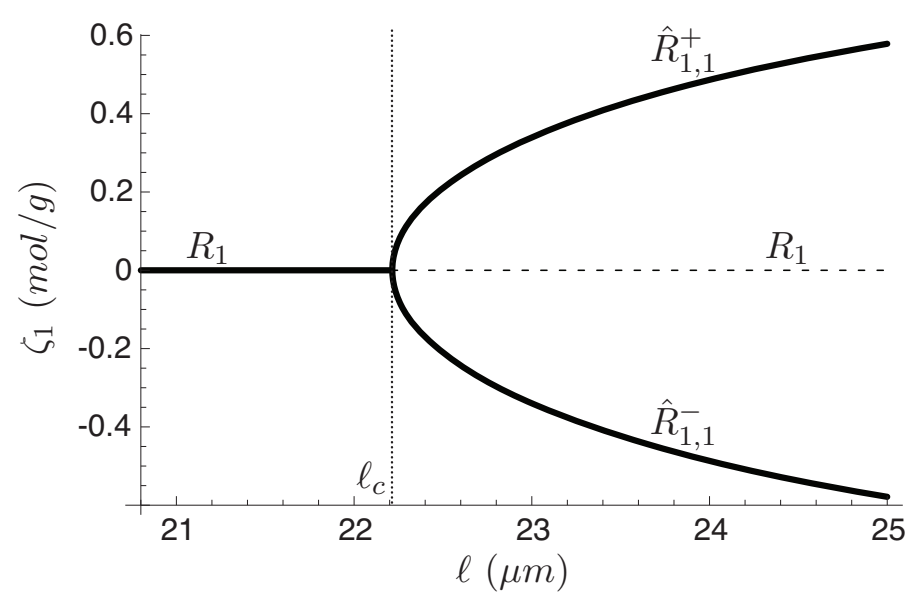

Figure 3. Locus of real equilibria in the neighborhood of $R_{1}$ for $B+B \leftrightharpoons A+A$.

therefore seek other candidate equilibria of the steady state Galerkin projection, (3.3), to fill the role of SIM-BIC for $\ell>\ell_{c}$.

When the domain length is an integer multiple of the critical length scale, $\ell=m \ell_{c},(4.13)$ predicts one of the spectra of eigenvalues at $R_{1}$ to be equal to zero. At these length scales, we numerically identify supercritical pitchfork bifurcations at $R_{1}$; two additional equilibria undergo a transition from complex to real through the spatially homogeneous equilibrium $R_{1}$. These equilibria have spatial symmetry, so we label them $\hat{R}_{1, m}^{+}$and $\hat{R}_{1, m}^{-}$. This bifurcation can be seen in Figure 3 for $m=1$, where the bold branches have one positive eigenvalue and the dashed branch has two. Since the dynamics of $\hat{R}_{1,1}^{+}$and $\hat{R}_{1,1}^{-}$are identical, as shown in section 3 , we can focus our analysis on one root, $\hat{R}_{1,1}^{+}$(with $\zeta_{1}>0$ ), and for simplicity drop the plus sign superscript. For $\ell>\ell_{c}$ the $\hat{R}_{1,1}$ equilibria each have one positive eigenvalue and are candidates for the SIM-BIC.

We examine the solution whose initial conditions are in the neighborhood of $\hat{R}_{1,1}$, perturbed along the eigenfunction whose eigenvalue is positive, and find that it approaches the physical equilibrium along its slowest spatially homogeneous eigenfunction. (Note that an eigenvector of the Jacobian of (3.3) approximates an eigenfunction of (3.1) and is referred to as such.) Therefore, the $\hat{R}_{1,1}$ equilibria meet the requirements to formally become SIM-BICs for all $\ell>\ell_{c}$.

Subsequent bifurcations at longer domain lengths exhibit similar properties; additional equilibria pairs, $\hat{R}_{1, m}$, correspond to bifurcations at integer multiples of the critical length scale. The bifurcation at $\ell=m \ell_{c}$ corresponds to the $m$ th Fourier mode's wavelength being identical to the critical length scale. Linear stability analysis of these equilibria shows that for $\ell>m \ell_{c}, \hat{R}_{1, m}$ will have only $m$ positive eigenvalues, and the remaining spectrum of eigenvalues will be negative.

Each $\hat{R}_{1, m}$ equilibrium is a fixed point on the finite-dimensional AIM that converges to an equilibrium manifold in the limit as $M \rightarrow \infty$. To show the convergence for $\hat{R}_{1,1}$, we quantify the error by comparing the steady solution in a lower-order truncation to its corresponding solution in an $M=25$ Galerkin projection and find the relative root mean square error, $\mathcal{E}_{i}$, 


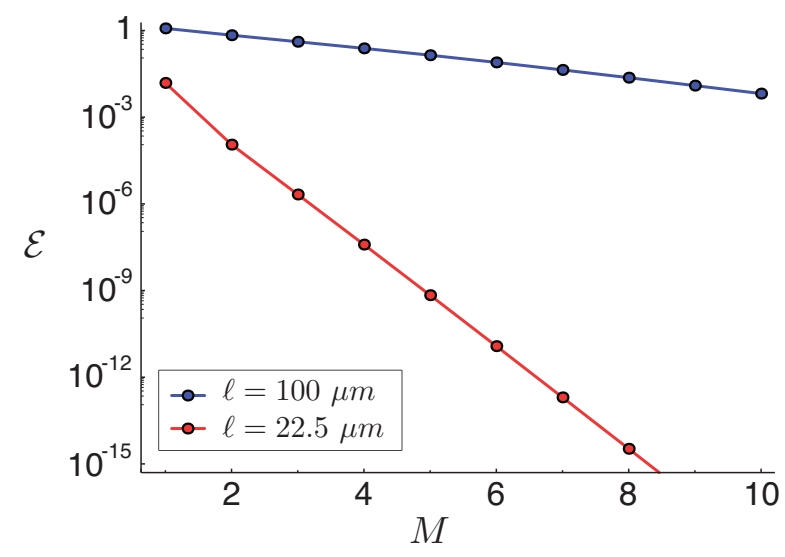

Figure 4. Relative error of $\hat{R}_{1,1}$ approximations for $B+B \leftrightharpoons A+A$.

which is defined as

$$
\mathcal{E}_{i}=\left(\frac{\int_{0}^{\ell}\left(\tilde{z}_{i}^{e}-\tilde{z}_{25}^{e}\right)^{2} d x}{\int_{0}^{\ell}\left(\tilde{z}_{25}^{e}\right)^{2} d x}\right)^{\frac{1}{2}} \quad \text { for } i=1, \ldots, 24 .
$$

This convergence is shown in Figure 4 for $\ell=22.5 \mu \mathrm{m}$ and $\ell=100 \mu \mathrm{m}$. The error of the spatially inhomogeneous steady state solution provides a good metric for the convergence of the Galerkin projection at a particular length scale, without imposing an arbitrary initial condition or requiring time integration. We see that the error for $\ell=100 \mu \mathrm{m}$ converges slowly, remaining relatively large, while the error for $\ell=22.5 \mu \mathrm{m}$ converges rapidly. This shows that for short domain lengths, where $\ell \lesssim \mathcal{O}\left(\ell_{c}\right)$, lower-order truncations not only provide accurate representation of the equilibrium manifold but also accurately describe the reaction-diffusion dynamics.

We now evaluate approximations of (4.11) with lower-order Galerkin projections. In the limit of an infinitesimal domain length, $\ell \downarrow 0$, the diffusion terms in the projection become infinitely fast for all $m>0$, any spatial inhomogeneities equilibrate immediately, and the system can be modeled as spatially homogeneous,

$$
\frac{d z}{d t}=-1.5 \times 10^{6}(z+1)\left(z-\frac{1}{3}\right) .
$$

In addition to $R_{1}$ and $R_{2}$, this system also has two infinite equilibria, $z^{e: S H} \rightarrow+\infty \mathrm{mol} / \mathrm{g}$, labeled $I_{1}$, and $z^{e: S H} \rightarrow-\infty \mathrm{mol} / \mathrm{g}$, labeled $I_{2}$. These infinite equilibria are identified using a Poincaré sphere mapping [48]; more details can be found in Appendix C. Evaluating the infinite equilibria, we find that $I_{1}$ is unstable and $I_{2}$ is stable. The rate of change, $\dot{\Omega}$, is plotted in Figure 5; the physical domain, where both species have positive concentrations, is shown as a gray-shaded region. For this $R=1$-dimensional spatially homogeneous system, a one-dimensional SIM is degenerate; however, we construct it to provide an example of how diffusion modifies the dynamics of a spatially homogeneous system. In one-dimensional systems, there are only sinks and sources, so the SIM-BIC in this case will be a source since

Copyright (C) by SIAM. Unauthorized reproduction of this article is prohibited. 


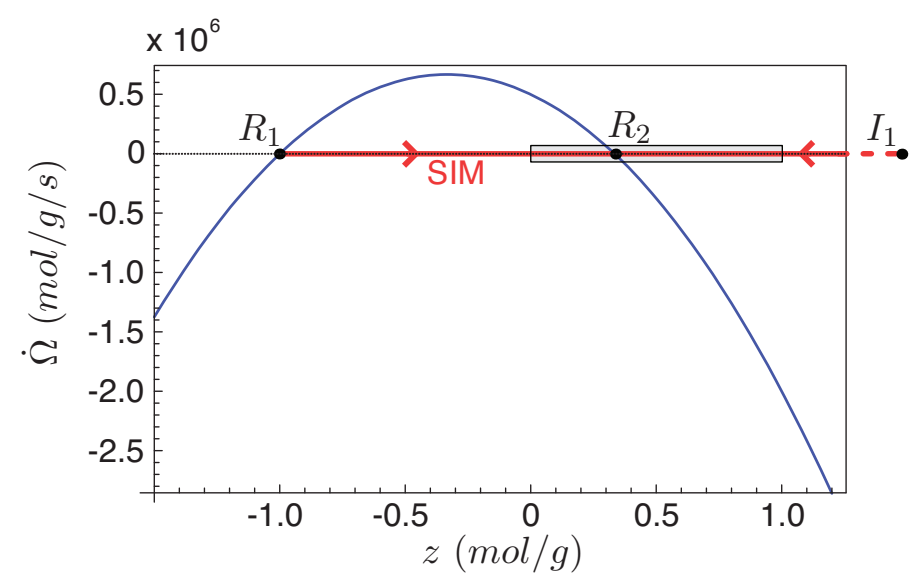

Figure 5. Spatially homogeneous rate of change, equilibria, and SIM branches for $B+B \leftrightharpoons A+A$.

(a)
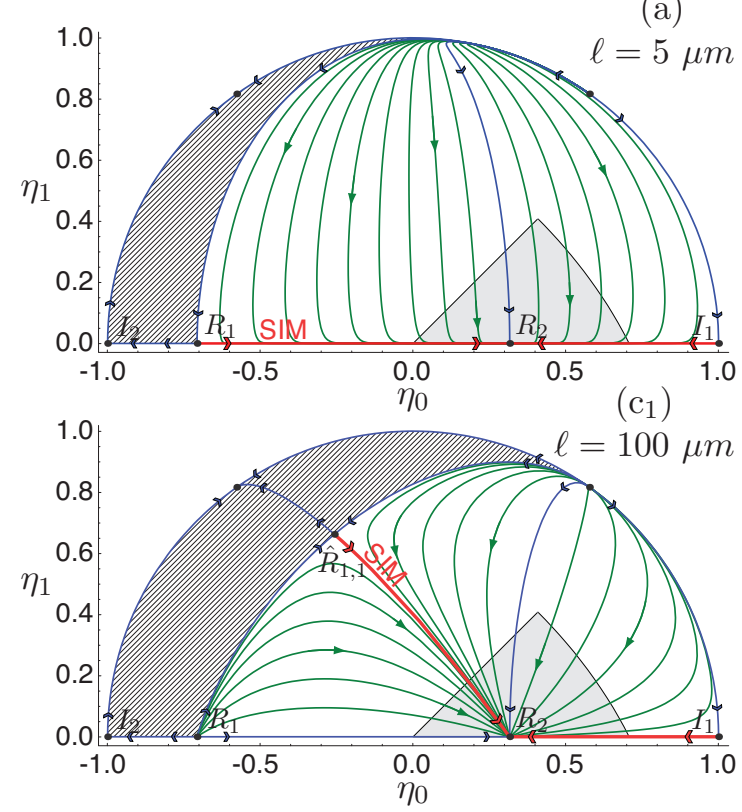

(b)

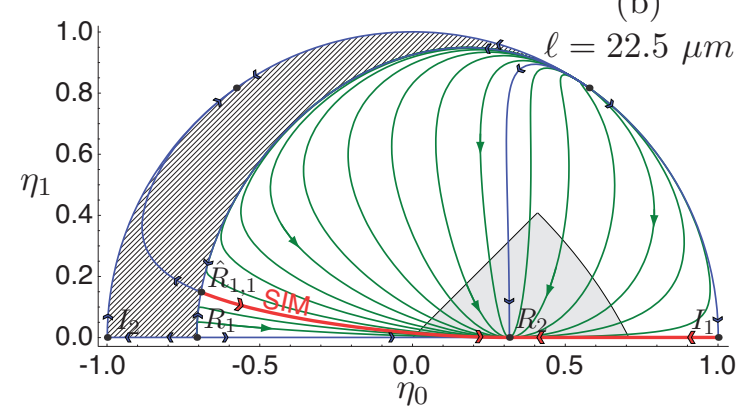

$\left(\mathrm{c}_{2}\right)$

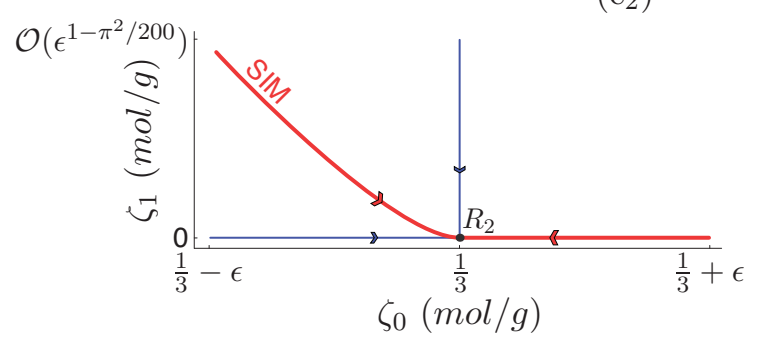

Figure 6. Poincaré sphere projection showing the $M=1$ Galerkin dynamics of $B+B \leftrightharpoons A+A$.

it must have at least one positive eigenvalue. To construct a branch of the SIM, we integrate a system trajectory from the SIM-BIC to the physical equilibrium sink. The two SIM-BICs for this system are at $R_{1}, z^{e}=-1$, and at $I_{1}$, positive infinity; the SIM branches are shown as a bold red line in Figure 5 and constitute the entire phase space between these SIM-BIC equilibria and the physical sink. We will focus our analysis of the reaction-diffusion system on the finite branch of the SIM between $R_{1}$ and $R_{2}$.

We now examine the performance of the spatially inhomogeneous diffusion-modified onedimensional SIM. In order to evaluate these roots in the entire phase space, we use a Poincaré sphere mapping [48]. Figure 6 shows a projection of the Poincaré sphere mapping of the 
$M=1$ truncation at three domain lengths which demonstrate the bifurcation of the SIM-BIC and the resulting changes in dynamics, where, as shown in Appendix C, $\eta_{0}$ and $\eta_{1}$ correspond to $\zeta_{0}$ and $\zeta_{1}$, respectively. In Figure 6(a), where $\ell=5 \mu m<\ell_{c}$, the diffusion time scale is much faster than the reaction time scale. At this length scale the SIM-BIC still resides at the spatially homogeneous $R_{1}$, whose eigenvalues are $\lambda=\left\{2 \times 10^{6},-3.748 \times 10^{7}\right\} \mathrm{s}^{-1}$. This causes the slow dynamics to be driven by the reaction mechanism only, and therefore the spatially homogeneous SIM remains the SIM for this reaction-diffusion system. Because the diffusion time scale is faster than the reaction time scale at this length, the trajectories all rapidly collapse onto the $\eta_{0}$ axis, the spatially homogeneous subspace. This results in the spatially homogeneous SIM accurately describing the long time dynamics of the system.

When the length scale is increased to $\ell=22.5 \mu \mathrm{m}>\ell_{c}$, as seen in Figure 6(b), the diffusion time scale is marginally slower than the unstable reaction time scale at $R_{1}$. At this domain length, the first pitchfork bifurcation has three real branches, $R_{1}$ and both $\hat{R}_{1,1}$ roots. The slower diffusion means it does not provide a sufficient stabilizing effect on $\lambda_{1,1}$, so $R_{1}$ has two positive eigenvalues, $\lambda=\left\{2 \times 10^{6}, 5.045 \times 10^{4}\right\} \mathrm{s}^{-1}$, and is therefore not a candidate for SIMBIC; however, $\hat{R}_{1,1}$ each have only one positive eigenvalue, $\lambda=\left\{2.047 \times 10^{6},-9.734 \times 10^{4}\right\} \mathrm{s}^{-1}$, and they formally assume the role as SIM-BIC; however, when we consider the eigenvalues of the physical equilibrium, $R_{2}, \lambda=\left\{-2 \times 10^{6},-3.950 \times 10^{6}\right\} s^{-1}$, we find that they have become much closer to the same order of magnitude. This lack of spectral gap indicates that the trajectories in the $\ell=22.5 \mu \mathrm{m}$ case will not collapse onto either one-dimensional SIM as quickly as they do for shorter lengths. In fact, for lengths on the order of and longer than $\ell_{c}$, trajectories define two- or higher-dimensional manifolds that describe the long time dynamics. Therefore, both the spatially homogeneous and diffusion-modified one-dimensional SIMs in Figure 6(b) appear less attractive than the SIM in Figure 6(a), where the trajectories in the latter figure approach the one-dimensional SIM with higher curvature than the former.

We consider a still longer length scale, $\ell=100 \mu m$, as seen in Figure 6( $\left.c_{1}\right)$. At this length, the $m=1$ diffusion time scale is too slow to provide an appreciable stabilizing effect on $\lambda_{1,1}$, and $R_{1}$ has two nearly identical positive eigenvalues, $\lambda=\left\{2 \times 10^{6}, 1.9013 \times 10^{6}\right\} \mathrm{s}^{-1} . \hat{R}_{1,1}$ remains the formal SIM-BIC for this length scale with eigenvalues $\lambda=\left\{2.048 \times 10^{6},-1.949 \times\right.$ $\left.10^{6}\right\} s^{-1}$. The ratio of the two eigenvalues associated with the slowest modes in the neighborhood of the physical equilibrium, $R_{2}$, approaches unity at this length scale, and, therefore, trajectories in this region do not collapse onto the one-dimensional SIM; however, the trajectories in the neighborhood of the SIM-BIC, $\hat{R}_{1}$, collapse onto the SIM with higher curvature than they did for the $\ell=22.5 \mu \mathrm{m}$ case. This is because the magnitude of the stable eigenvalue is on the same order of magnitude as the unstable eigenvalue, making for a saddle whose trajectories collapse onto the unstable eigenvector. The SIM in Figure 6( $\left.\mathrm{c}_{1}\right)$ does not appear to approach $R_{2}$ along the slowest (spatially homogeneous) eigenvector, but upon a linear evaluation of the SIM in the neighborhood of $R_{2}$, a point on the SIM at $\zeta_{0}=1 / 3-\epsilon$, it will have $\zeta_{1} \sim \mathcal{O}\left(\epsilon^{1-\pi^{2} / 200}\right)$ as $\epsilon$ decays to zero, where the exponent, $1-\pi^{2} / 200$, is just less than 1. Therefore, as shown in Figure $6\left(\mathrm{c}_{2}\right)$, the SIM osculates the $\zeta_{0}$ axis in its approach to $R_{2}$, confirming that this manifold meets all of our SIM criteria. The $M=1$ truncation at this length scale is underresolved; however, the dynamics presented in Figure 6(c) are qualitatively the same as a well-resolved truncation.

This analysis shows that the use of the spatially homogeneous SIM is valid only for $\ell<\ell_{c}$;

Copyright (c) by SIAM. Unauthorized reproduction of this article is prohibited. 
when $\ell>\ell_{c}$, the bifurcation indicates that both reaction and diffusion must be accounted for in order for a reduced manifold to potentially capture the slow dynamics of the system. Furthermore, a spectral gap needs to be present for a manifold to attract local trajectories and accurately capture the slow dynamics; for $\ell>\ell_{c}$, a one-dimensional SIM proves to be inadequate.

4.2. Zel'dovich mechanism. In this section we examine the Zel'dovich mechanism of NO production, which is shown in Table 1 . We then show the reduction of the stoichiometric constraints, examine the spatially homogeneous system, identify the critical length where the SIM-BIC bifurcates, compare local reaction and diffusion time scales, and illustrate the effect of length scales on the trajectories in the $M=1$ Galerkin projection phase space.

Table 1

The Zel'dovich mechanism for NO production.

\begin{tabular}{cccc}
\hline Reaction & $\begin{array}{c}a_{j} \\
{\left[\mathrm{~cm}^{3} /(\mathrm{mol} \mathrm{s}) \mathrm{K}^{-\beta_{j}}\right]}\end{array}$ & $\beta_{j}$ & $\begin{array}{c}\bar{E}_{j} \\
{[\mathrm{erg} / \mathrm{mol}]}\end{array}$ \\
\hline$N+\mathrm{O}_{2} \leftrightharpoons \mathrm{NO}+\mathrm{O}$ & $5.841 \times 10^{9}$ & 1.01 & 6195.6 \\
$N+N O \leftrightharpoons N_{2}+O$ & $21.077 \times 10^{12}$ & 0.00 & 0.0 \\
\hline
\end{tabular}

Al-Khateeb et al. [18] studied the spatially homogeneous Zel'dovich mechanism under isothermal and isochoric constraints; we extend their analysis to reaction-diffusion systems. This system displays realistic chemical dynamics and has a slightly higher dimension $(R=2)$ than the previous example. There are $N=5$ species, $J=2$ reactions, and $L=2$ elements, where the species $\left\{N O, N, O, O_{2}, N_{2}\right\}$ correspond to $i=1, \ldots, 5$. The species-reaction matrix for this mechanism is

$$
\nu_{i j}=\left[\begin{array}{rr}
1 & -1 \\
-1 & -1 \\
1 & 1 \\
-1 & 0 \\
0 & 1
\end{array}\right]
$$

and the species-element matrix is

$$
\varphi_{l i}=\left[\begin{array}{ccccc}
1 & 1 & 0 & 0 & 2 \\
1 & 0 & 1 & 2 & 0
\end{array}\right]
$$

The reaction rates are given by

$$
\begin{aligned}
& r_{2}=k_{2}\left(\frac{\rho Y_{2}}{\overline{\mathrm{M}}_{N}} \frac{\rho Y_{4}}{\overline{\mathrm{M}}_{O_{2}}}-\frac{1}{K_{2}^{c}} \frac{\rho Y_{1}}{\overline{\mathrm{M}}_{N O}} \frac{\rho Y_{3}}{\overline{\mathrm{M}}_{O}}\right), \\
& r_{1}=k_{1}\left(\frac{\rho Y_{2}}{\overline{\mathrm{M}}_{N}} \frac{\rho Y_{1}}{\overline{\mathrm{M}}_{N O}}-\frac{1}{K_{1}^{c}} \frac{\rho Y_{5}}{\overline{\mathrm{M}}_{N_{2}}} \frac{\rho Y_{3}}{\overline{\mathrm{M}}_{O}}\right) .
\end{aligned}
$$

This mechanism has one additional constraint; since both reactions are bimolecular, the total 
number of molecules remains constant; therefore, the system has $\hat{L}=3$ algebraic constraints,

$$
\begin{aligned}
C_{M} & =\frac{Y_{1}}{\overline{\mathrm{M}}_{N O}}+\frac{Y_{2}}{\overline{\mathrm{M}}_{N}}+\frac{Y_{3}}{\overline{\mathrm{M}}_{O}}+\frac{Y_{4}}{\overline{\mathrm{M}}_{O_{2}}}+\frac{Y_{5}}{\overline{\mathrm{M}}_{N_{2}}}, \\
C_{N} & =\frac{Y_{1}}{\overline{\mathrm{M}}_{N O}}+\frac{Y_{2}}{\overline{\mathrm{M}}_{N}}+2 \frac{Y_{5}}{\overline{\mathrm{M}}_{N_{2}}}, \\
C_{O} & =\frac{Y_{1}}{\overline{\mathrm{M}}_{N O}}+\frac{Y_{3}}{\overline{\mathrm{M}}_{O}}+2 \frac{Y_{4}}{\overline{\mathrm{M}}_{O_{2}}} .
\end{aligned}
$$

The variables $C_{M}, C_{N}$, and $C_{O}$ are constants that are defined by the initial conditions and correspond to the conservation of total molecules, nitrogen atoms, and oxygen atoms, respectively. We use these algebraic constraints to transform our system into two reduced variables, choosing specific moles of $N O$ and $N$, which we represent as $z_{1}$ and $z_{2}$, respectively,

$$
\begin{aligned}
& z_{1}=\frac{Y_{1}}{\mathrm{M}_{N O}}, \\
& z_{2}=\frac{Y_{2}}{\mathrm{M}_{N}} .
\end{aligned}
$$

Using the reduction technique from section 2.3, we find the modified coefficient matrix to be

$$
\mathcal{D}_{i n}=\left[\begin{array}{rr}
1 & 0 \\
0 & 1 \\
0 & -1 \\
-\frac{1}{2} & \frac{1}{2} \\
-\frac{1}{2} & -\frac{1}{2}
\end{array}\right] .
$$

The remaining $\hat{L}=3$ species specific mole values are coupled to $z_{1}$ and $z_{2}$ by the algebraic relations

$$
\begin{aligned}
& \hat{z}_{3}=\hat{z}_{3}^{*}+z_{2}^{*}-z_{2}, \\
& \hat{z}_{4}=\hat{z}_{4}^{*}+\frac{1}{2} z_{1}^{*}-\frac{1}{2} z_{2}^{*}-\frac{1}{2} z_{1}+\frac{1}{2} z_{2}, \\
& \hat{z}_{5}=\hat{z}_{5}^{*}+\frac{1}{2} z_{1}^{*}+\frac{1}{2} z_{2}^{*}-\frac{1}{2} z_{1}-\frac{1}{2} z_{2} .
\end{aligned}
$$

This system is evaluated at the constant temperature, $T=4000 K$, and density, $\rho=$ $1.20024 \times 10^{-4} \mathrm{~g} / \mathrm{cm}^{3}$, which yields a constant pressure, $P=1.64 \mathrm{~atm}$. The species' thermodynamic data comes from the CHEMKIN thermodynamic database [49]. The diffusion coefficient, $D=14.0 \mathrm{~cm}^{2} / \mathrm{s}$, is approximated from a weighted average of the ordinary multicomponent coefficients in the CHEMKIN TRANSPORT database [50]. We choose initial conditions such that the values of the constants are $C_{O}=C_{N}=3.3327 \times 10^{-2} \mathrm{~mol} / \mathrm{g}$ and $C_{M}=4.1658 \times 10^{-2} \mathrm{~mol} / \mathrm{g}$. The governing equations, when transformed into specific moles, are

$$
\begin{aligned}
& \frac{\partial z_{1}}{\partial t}=C_{10}+C_{11} z_{1}+C_{12} z_{2}+C_{13} z_{1} z_{2}+C_{14} z_{2}^{2}+D \frac{\partial^{2} z_{1}}{\partial x^{2}} \\
& \frac{\partial z_{2}}{\partial t}=C_{20}+C_{21} z_{1}+C_{22} z_{2}+C_{23} z_{1} z_{2}+C_{24} z_{2}^{2}+D \frac{\partial^{2} z_{1}}{\partial x^{2}}
\end{aligned}
$$

Copyright (C) by SIAM. Unauthorized reproduction of this article is prohibited. 
Table 2

Values of the coefficients, $C_{i j}$, for Zel'dovich mechanism evolution equations.

\begin{tabular}{cccccr}
\hline & $j=0$ & $j=1$ & $j=2$ & $j=3$ & \multicolumn{1}{c}{$j=4$} \\
& {$\left[\frac{\mathrm{mol}}{\mathrm{gs}}\right]$} & {$\left[\frac{1}{s}\right]$} & {$\left[\frac{1}{\mathrm{~s}}\right]$} & {$\left[\frac{\mathrm{g}}{\mathrm{mol}}\right]$} & \multicolumn{1}{c}{$\left[\frac{\mathrm{g}}{\mathrm{mol} \mathrm{s}}\right]$} \\
\hline$i=1$ & 250.46 & -99728 & $1.1611 \times 10^{7}$ & $-3.2210 \times 10^{9}$ & $6.9858 \times 10^{8}$ \\
$i=2$ & 250.46 & 84697 & $-1.1656 \times 10^{7}$ & $-1.8359 \times 10^{9}$ & $-6.9768 \times 10^{8}$ \\
\hline
\end{tabular}
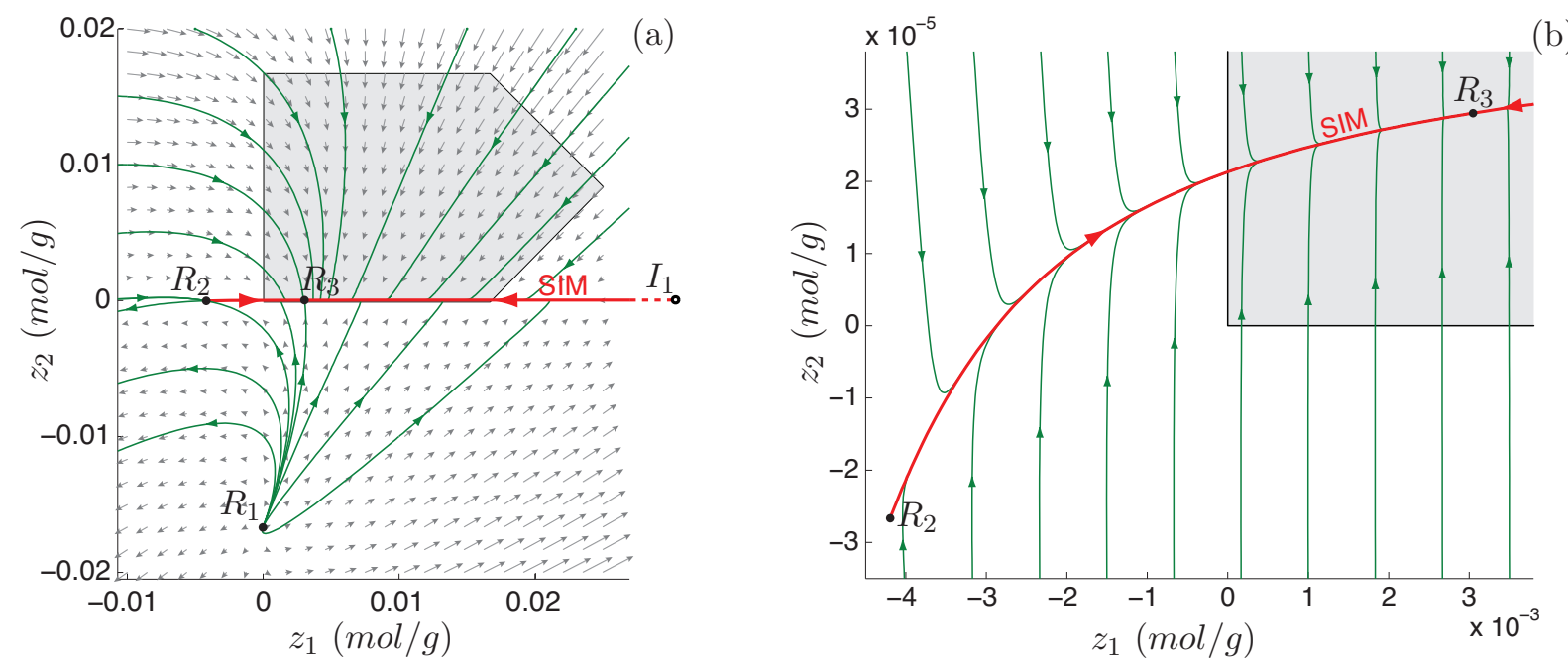

Figure 7. The spatially homogeneous SIM for the Zel'dovich mechanism.

where the coefficients, $C_{i j}$, are given in Table 2 .

When we evaluate the system in the limit of an infinitesimal domain length, a truncation at $M=0$ is appropriate, and we are left with the spatially homogeneous system,

$$
\begin{aligned}
& \frac{d z_{1}}{d t}=C_{10}+C_{11} z_{1}+C_{12} z_{2}+C_{13} z_{1} z_{2}+C_{14} z_{2}^{2}, \\
& \frac{d z_{2}}{d t}=C_{20}+C_{21} z_{1}+C_{22} z_{2}+C_{23} z_{1} z_{2}+C_{24} z_{2}^{2},
\end{aligned}
$$

which is the same system described by Al-Khateeb et al. [18]. The phase space for this system is shown in Figure 7. This system has three finite spatially homogeneous equilibria,

$$
\mathbf{z}^{e}=\left\{\left[\begin{array}{l}
-1.7833 \times 10^{-5} \\
-1.6681 \times 10^{-2}
\end{array}\right],\left[\begin{array}{l}
-4.1950 \times 10^{-3} \\
-2.6642 \times 10^{-5}
\end{array}\right],\left[\begin{array}{l}
3.0474 \times 10^{-3} \\
2.9446 \times 10^{-5}
\end{array}\right]\right\} \mathrm{mol} / \mathrm{g},
$$

labeled $R_{1}, R_{2}$, and $R_{3}$, respectively. The eigenvalues of the linearized system in the neighborhood of each equilibrium are near $R_{1}, \lambda=\left\{4.1760 \times 10^{7}, 2.3523 \times 10^{7}\right\} \mathrm{s}^{-1}$; near $R_{2}$, $\lambda=\left\{7.1039 \times 10^{5},-4.6413 \times 10^{6}\right\} \mathrm{s}^{-1}$; and near $R_{3}, \lambda=\left\{-1.9129 \times 10^{5},-1.7295 \times 10^{7}\right\} \mathrm{s}^{-1}$. $R_{3}$ is the physical equilibrium sink, while the nonphysical $R_{1}$ and $R_{2}$ are of source and saddle types, respectively; we find that $R_{2}$ is a SIM-BIC. The second branch of the SIM originates at a SIM-BIC at $z \rightarrow+\infty, I_{1}$. We focus our analysis on the SIM that connects $R_{2}$ to $R_{3}$. Figure 7 (a) shows the physically realizable region shaded gray, individual rate of change vectors as

Copyright $\odot$ by SIAM. Unauthorized reproduction of this article is prohibited. 


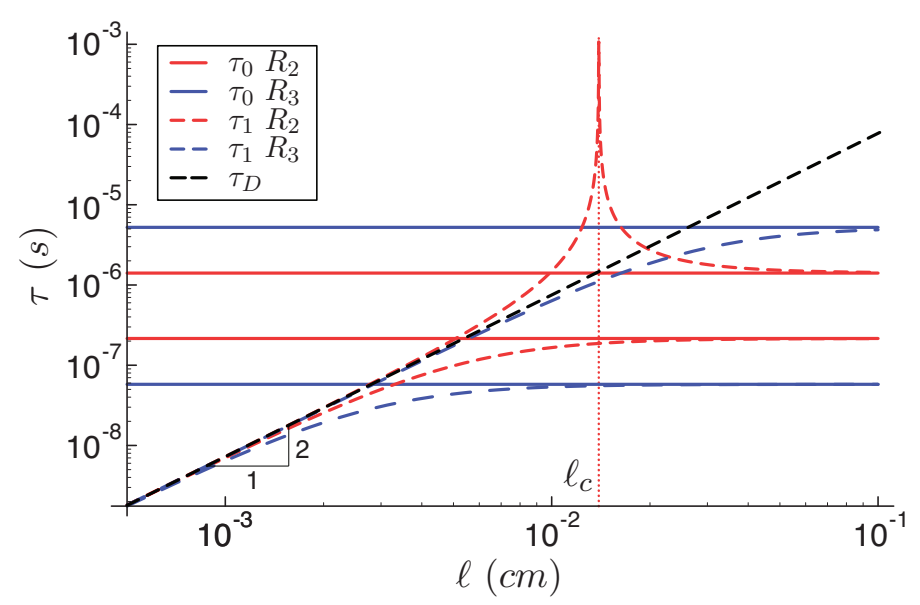

Figure 8. Local time scales of $R_{2}$ and $R_{3}$ for the Zel'dovich mechanism.

arrows, system trajectories as thin green lines, and the SIM's two branches as bold red lines. In this system we see a disparity in the two reaction time scales, resulting in high curvature of trajectories as they approach the SIM, which is shown in the close-up plot of the SIM in Figure 7(b).

We now evaluate the Galerkin projection to predict the effects of diffusion across finite length scales. Similar to the results from the example in section 4.1, this system retains the spatially homogeneous equilibria and gains (among others) pairs of equilibria, $\hat{R}_{2, m}$, that bifurcate from the $R_{2}$ equilibria at integer multiples of a critical length scale,

$$
\ell_{c}=\pi \sqrt{\frac{D}{\lambda_{0,1}^{e: R 2}}}=139.5 \mu \mathrm{m} .
$$

This critical length scale is the domain length at which the $m=1$ diffusion time scale equals the unstable reaction time scales at $R_{2}$; this once again demonstrates that diffusion processes can couple chemical time scales to length scales: $\ell_{c}=\pi \sqrt{D \tau_{0}}$. The bifurcations at $\ell_{c}$ are of supercritical pitchfork type, where $\hat{R}_{2, m}$ are real only for domain lengths longer than $m \ell_{c}$. Linear analysis in the neighborhood of $\hat{R}_{2, m}$ reveals each equilibrium has $m$ positive eigenvalues; in the neighborhood of $R_{2}$ there are $m$ positive eigenvalues when the domain length is between $(m-1) \ell_{c}<\ell<m \ell_{c}$. We note that $R_{2}$ will have a zero eigenvalue for domains that are an integer multiple of $\ell_{c}$ as an eigenvalue changes from negative to positive; these zero eigenvalues correspond to the bifurcations. This can be seen for $m=1$ in Figure 8, which shows the time scales in the neighborhoods of $R_{2}$ and $R_{3}$ as a function of domain length, for the $m=0$ and $m=1$ modes. For the $m=1$ time scales, we see that short domain lengths are dominated by the diffusion contribution, while long domain lengths are dominated by the reaction contribution. Where the transition occurs, there is a singularity associated with the zero eigenvalue and the bifurcation at $R_{2}$; this occurs at $\ell=\ell_{c}$. Similar results for larger values of $m$ occur at subsequent bifurcations. To evaluate the quality of the Galerkin projection, a convergence study was performed. The convergence rates for the $\hat{R}_{2,1}$ equilibria are similar to those shown in Figure 4: for short domain lengths, $\ell \sim \mathcal{O}\left(\ell_{c}\right)$, the approximation converges

Copyright (c) by SIAM. Unauthorized reproduction of this article is prohibited. 
rapidly, while for longer domain lengths, $\ell>\ell_{c}$, it converges more slowly.

We now examine the dynamics of the reaction-diffusion system at five length scales, $\ell=$ $8.96 \mu \mathrm{m}, 22.4 \mu \mathrm{m}, 56 \mu \mathrm{m}, 140 \mu \mathrm{m}$, and $350 \mu \mathrm{m}$, in the $M=1$ truncation of the Galerkin projection. For $\ell=8.96 \mu \mathrm{m}$, which is significantly less than $\ell_{c}$, the diffusion time scales are much faster than all of the reaction time scales. This is seen when we examine the eigenvalues in the neighborhood of $R_{2}, \lambda=\left\{7.1039 \times 10^{5},-4.6413 \times 10^{6},-1.7140 \times 10^{8},-1.7675 \times 10^{8}\right\} s^{-1}$. This leads to a system that essentially behaves like a spatially homogeneous system, which is demonstrated in Figure 9(a), where the initial spatial inhomogeneities decay rapidly, resulting in the spatially homogeneous reaction which dictates the long time dynamics.

For $\ell=22.4 \mu \mathrm{m}$, which is still much less than $\ell_{c}$, the diffusion time scales are still faster than all of the reaction time scales, which is evident when examining the eigenvalues in the neighborhood of $R_{2}, \lambda=\left\{7.1039 \times 10^{5},-4.6413 \times 10^{6},-2.6828 \times 10^{7},-3.2179 \times 10^{7}\right\} \mathrm{s}^{-1}$. Diffusion causes the spatial inhomogeneities to decay before substantial reaction occurs; however, as seen in Figure 9(b), some reaction processes occur before diffusion has removed the majority of the spatial inhomogeneities. While the reaction mechanism and spatially homogeneous SIM dictate the long time dynamics, diffusion plays a bigger role in the approach to the SIM.

The next length scale, $\ell=56 \mu \mathrm{m}$, is still less than $\ell_{c}$; however, the fundamental diffusion time scale is now marginally slower than the fast reaction time scales in the neighborhoods of the $R_{2}$ and $R_{3}$ equilibria; the eigenvalues at $R_{2}$ are $\lambda=\left\{7.1039 \times 10^{5},-4.6413 \times 10^{6},-3.6957 \times\right.$ $\left.10^{6},-9.0474 \times 10^{6}\right\} s^{-1}$. This system no longer behaves like a spatially homogeneous system since both reaction and diffusion are acting on the same time scales; however, the slowest dynamics are still captured by the spatially homogeneous SIM since reaction remains the slowest time scale. This is seen in Figure 9(c), where the initial spatial inhomogeneities do not decay onto the spatially homogeneous subspace, yet the long time dynamics collapse onto the spatially homogeneous SIM.

Consider $\ell=140 \mu \mathrm{m}$, which is just slightly longer than $\ell_{c}$; therefore, at $R_{2}$ there are now two positive eigenvalues, $\lambda=\left\{7.1039 \times 10^{5},-4.6413 \times 10^{6}, 5418.9,-5.3463 \times 10^{6}\right\} s^{-1}$. The bifurcated equilibrium, $\hat{R}_{2,1}$, is now real and has only one positive eigenvalue, $\lambda=$ $\left\{7.2371 \times 10^{5},-4.6078 \times 10^{6},-10540,-5.4259 \times 10^{6}\right\} \mathrm{s}^{-1}$. We predict changes in the slow dynamics of the system, as is evident in Figure 9(d). For each of the shorter domain lengths, the fast diffusion causes the spatial inhomogeneities to decay rapidly enough that the spatially homogeneous SIM dictates the long time dynamics of the system; however, at $\ell=\ell_{3}$, the spatial inhomogeneities persist through the duration of both reaction time scales. The diffusion-modified one-dimensional SIM for this length scale is shown in Figure 9(c); however, due to the similarities of the slow reaction and slow diffusion time scales, the long time dynamics of the system are likely better captured by a two-dimensional manifold. As the amplitudes of modes with fast time scales decay, the trajectories relax onto a vertical sheet, where slow reaction and slow diffusion both dictate the dynamics as the trajectory proceeds toward equilibrium.

For the longest length scale considered, $\ell=350 \mu \mathrm{m}$, the diffusion time scale of the fundamental Fourier mode is significantly slower than both reaction time scales. We see in Figure $9(\mathrm{e})$ that the bifurcated equilibrium, $\hat{R}_{2,1}$, has become more spatially inhomogeneous, and the system's dynamics reflect this change in trajectories with spatial variations that

Copyright (c) by SIAM. Unauthorized reproduction of this article is prohibited. 
(a)

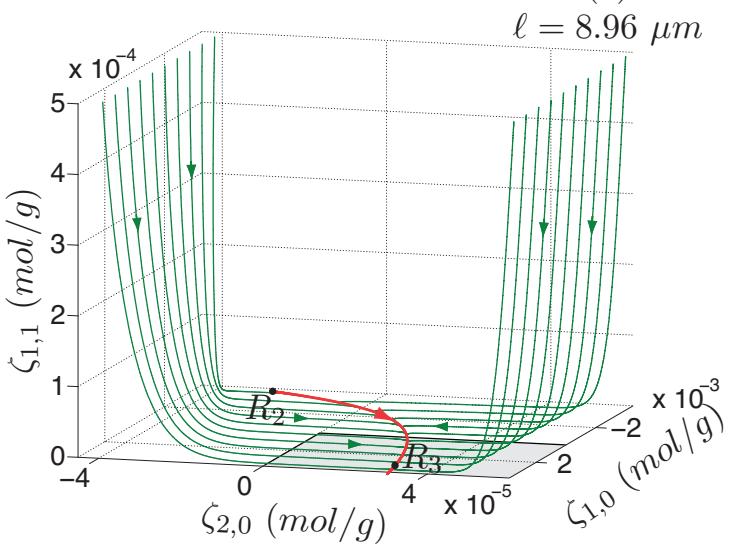

(b)

(c)
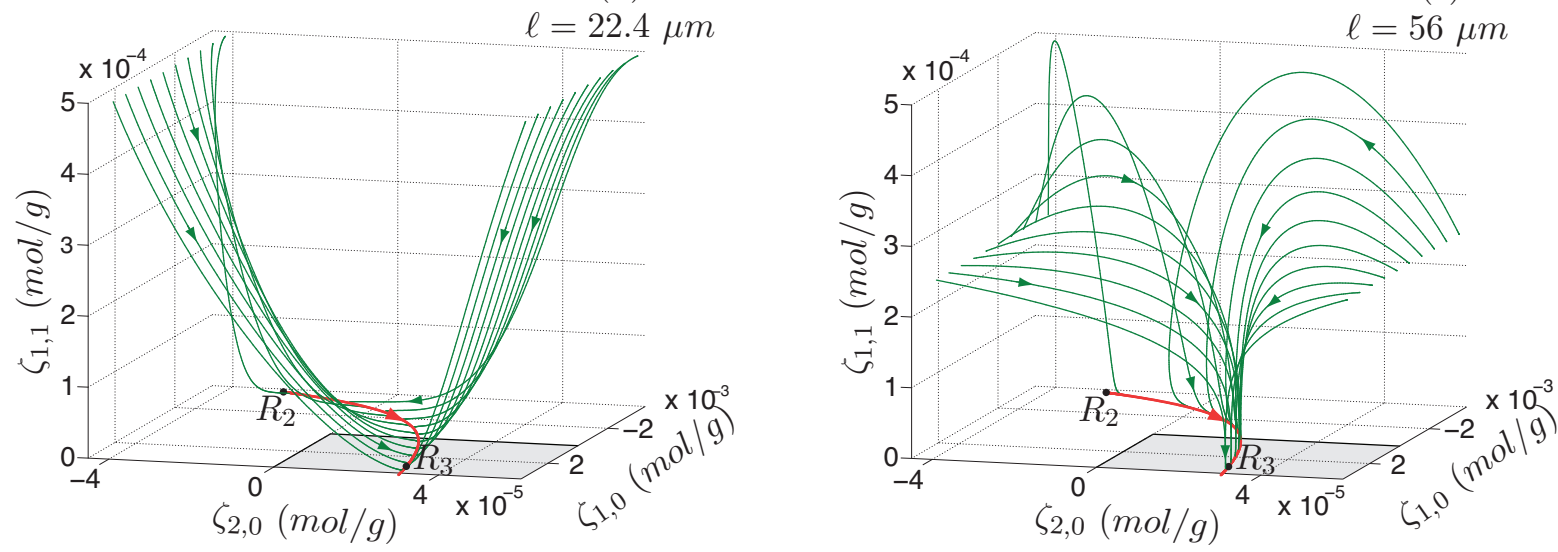

(d)

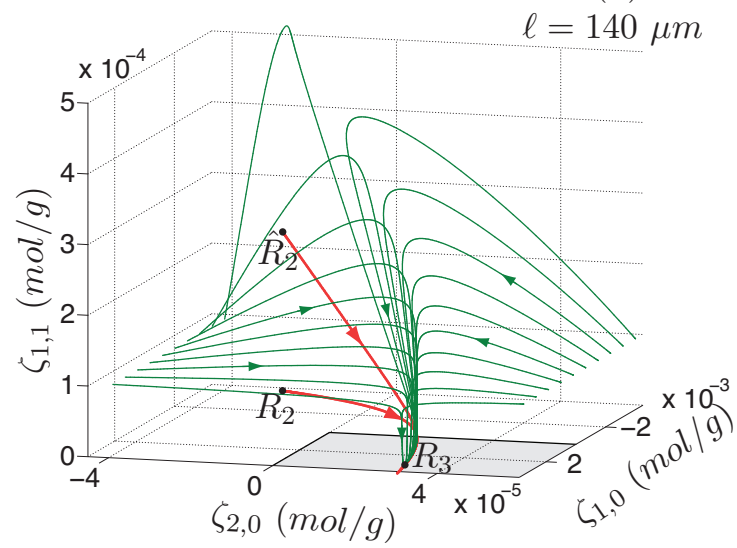

(e)

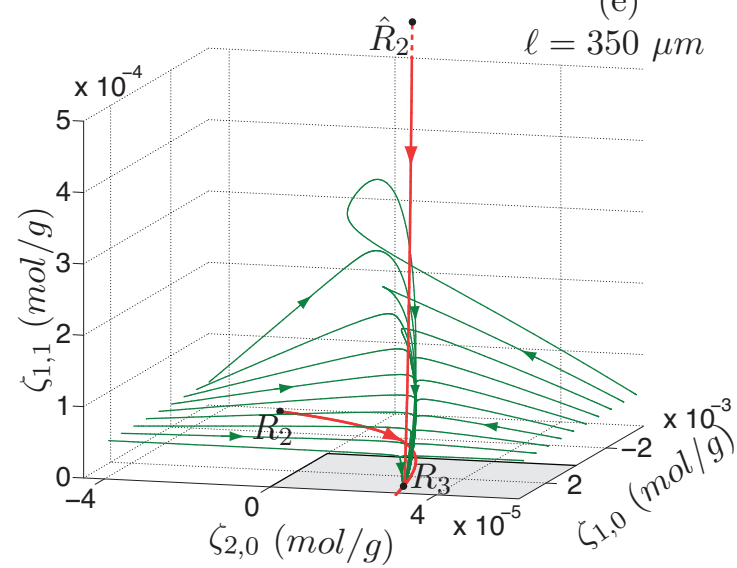

Figure 9. $M=1$ Galerkin projection phase space for the Zel'dovich mechanism at various $\ell$. 
(a)

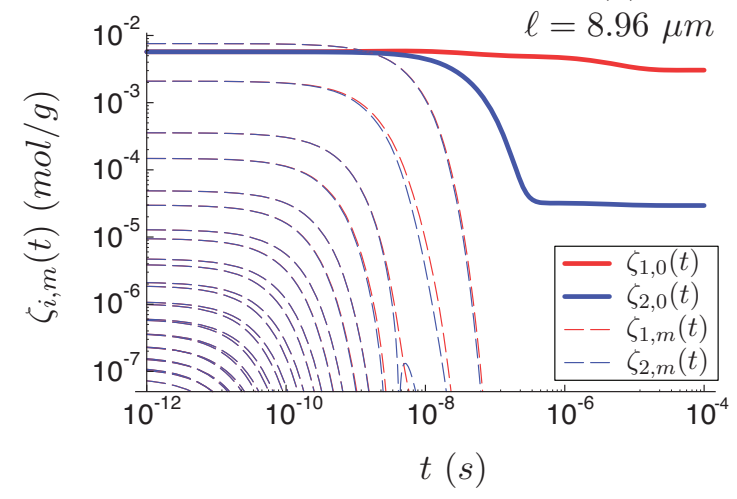

(c)

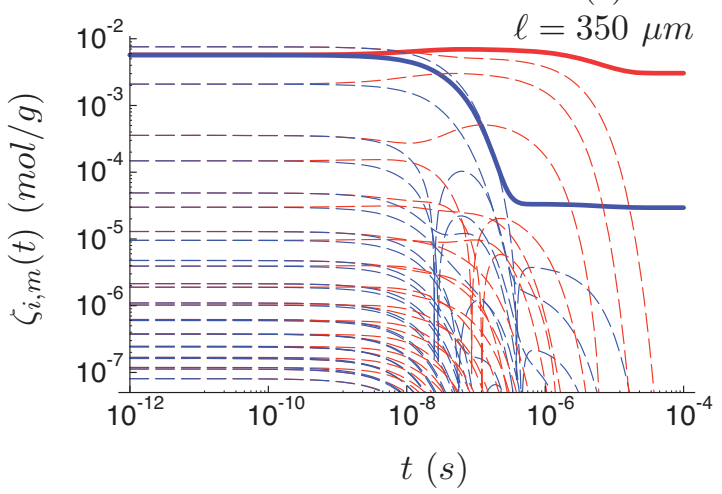

(b)

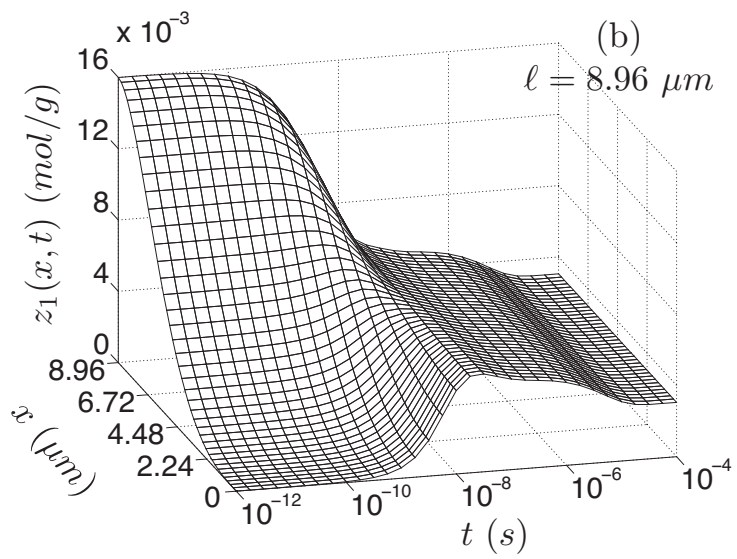

(d)

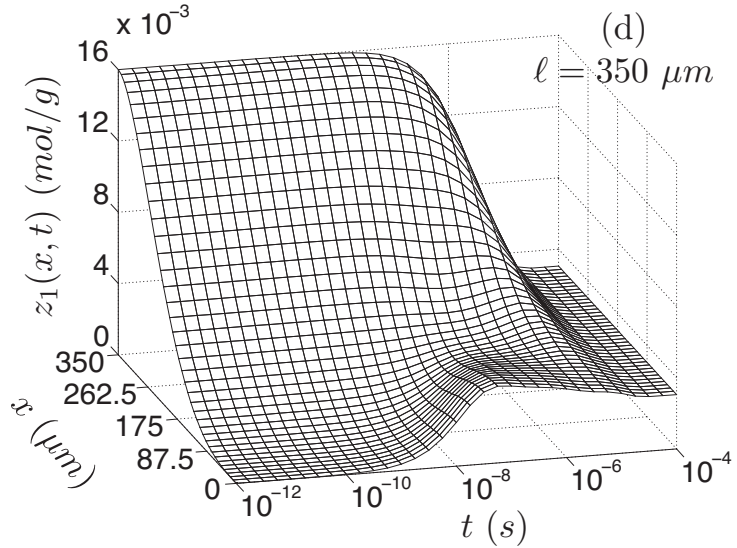

Figure 10. Amplitudes and spatial reconstruction of Zel'dovich mechanism species evolution in $M=25$ Galerkin projection.

persist further in the approach to equilibrium. There are still two unstable eigenvalues at $R_{2}, \lambda=\left\{7.1039 \times 10^{5},-4.6413 \times 10^{6}, 5.9760 \times 10^{5},-4.7541 \times 10^{6}\right\} \mathrm{s}^{-1}$, and one unstable eigenvalue at $\hat{R}_{2,1}, \lambda=\left\{9.3244 \times 10^{5},-4.2979 \times 10^{6},-2.1765 \times 10^{5},-1.3993 \times 10^{7}\right\} \mathrm{s}^{-1}$. Similar to the simple reaction mechanism, the diffusion-modified SIM appears to have the trajectories collapse onto it more than in the $\ell=140 \mu \mathrm{m}$ case, but again it requires more Galerkin modes to provide enough spatial resolution.

In Figure 10 we see the evolution of the two extreme length scales, $\ell=8.96 \mu \mathrm{m}(\mathrm{a}, \mathrm{b})$ and $\ell=350 \mu \mathrm{m}(\mathrm{c}, \mathrm{d})$, calculated in the $M=25$ Galerkin projection. The initial conditions for each are polynomials scaled to each domain length: $z_{1}^{*}(x)=\left(1.6 \times 10^{-2} \mathrm{~mol} / \mathrm{g}\right)\left(2(x / \ell)^{3}-\right.$ $\left.(x / \ell)^{6}\right)$ and $z_{2}^{*}(x)=\left(1.6 \times 10^{-2} \mathrm{~mol} / \mathrm{g}\right)\left(2(1-x / \ell)^{3}-(1-x / \ell)^{6}\right)$. On the left, Figure 10(a, c) shows the evolution of the amplitudes of $N O$ (red lines) and $N$ (blue lines), both spatially homogeneous (bold lines) and inhomogeneous (dashed lines). The spatial approximations of $N O$ evolution, reconstructed from (2.4), are shown on the right in Figure 10(b, d). Changes in the spatially homogeneous amplitudes in Figure 10(a, c) show the fast and slow reaction time scales, which occur at approximately $\tau_{1,0}=5.8 \times 10^{-8} \mathrm{~s}$ and $\tau_{2,0}=5.2 \times 10^{-6} \mathrm{~s}$. The diffusion time scales have drastic changes on the decay rate of the spatial inhomogeneity that is dependent on length: in the $\ell=8.96 \mu \mathrm{m}$ case, the diffusion time scale of the $m=1$

Copyright (C) by SIAM. Unauthorized reproduction of this article is prohibited. 
mode, $\tau_{D, 1}=5.8 \times 10^{-9} \mathrm{~s}$, is the fastest, which causes all spatial inhomogeneities to decay before either reaction time scale; in the $\ell=350 \mu \mathrm{m}$ case, the $m=1$ diffusion time scale, $\tau_{D, 1}=8.9 \times 10^{-6} \mathrm{~s}$, is the slowest, and large spatial inhomogeneities persist until the slow reaction time scale. The effects of the various diffusion time scales are also apparent in the spatial reconstruction: for $\ell=8.96 \mu \mathrm{m}$, diffusion causes the spatial inhomogeneities to decay rapidly, and the long time dynamics are governed by reaction only; for $\ell=350 \mu \mathrm{m}$, the long time dynamics display both reaction and diffusion processes.

We see that for domains that are shorter than the critical length scale, spatial inhomogeneities decay more rapidly than the amplitude of the spatially homogeneous mode with a slow reaction time scale, and the spatially homogeneous SIM describes the long time dynamics. For domains that are longer than the critical length scale, diffusion time scales are slower than the slow reaction time scale; therefore, in these longer domains the long time dynamics display spatial inhomogeneities, and the spatially homogeneous SIM is no longer appropriate to use as a reduction method.

5. Summary and conclusions. We have extended the robust method of SIM construction presented by Davis and Skodje [13] to reaction-diffusion systems. Through the use of a Galerkin projection, we analytically isolated the reaction and diffusion contributions to a modified time scale associated with each mode of a particular wavelength. We found a critical length scale at which the slowest diffusion time scale is equal to an unstable reaction time scale of the spatially homogeneous problem. At this critical length scale, a pitchfork bifurcation was predicted. When modeling any system with length longer than the critical value, the canonical slow manifold is no longer the spatially homogeneous SIM. While a onedimensional diffusion-modified SIM was constructed, it is not endowed with a wide spectral gap in its slowest time scales, thus rendering its utility to be of limited value in a reduction strategy for large scale realistic systems of engineering relevance. We have examined only small reaction mechanisms with a limited number of Galerkin modes. However, we believe our conclusions will extend to arbitrarily sized systems. When this is done, be it via addition of more detailed reactions, more Galerkin modes, or more spatial dimensions, the complexity of implementing this manifold method in high-dimensional phase space will rapidly overwhelm most present analysis strategies. This presents a daunting challenge for rational reduction of realistic combustion systems.

Appendix A. Galerkin projection of polynomials with cosine basis functions. To reduce computational costs as well as simplify the analysis, the inner products of arbitrary low-order monomials are analytically simplified into their respective Galerkin projection amplitudes for cosine basis functions. This is relevant since our reaction source terms in isothermal Arrhenius kinetics are low-order polynomial systems, and the inner product distributes to each monomial as a linear operator. Here we examine up to third-order monomial expansions of arbitrary species labeled with subscripts $a, b$, and $c$.

In the following derivations, we take advantage of the fact that cosine is an even function,

$$
\cos \left(\frac{m \pi x}{\ell}\right)=\cos \left(-\frac{m \pi x}{\ell}\right) \text {. }
$$

We apply the normalized inner product for our orthogonal basis functions with arbitrarily

Copyright (c) by SIAM. Unauthorized reproduction of this article is prohibited. 
signed arguments by using the Kronecker delta function for both the positive and negative of our function's argument.

Since the inner product is a linear operator and can be distributed to each term of a polynomial, we examine each order of monomial individually. For constants, we see that the normalized inner product results in the constant appearing only in the $m=0$ term of the Galerkin projection. When $\phi_{0}=1$, as it does in our Galerkin projection, the constant remains unchanged,

$$
\frac{\left\langle\phi_{m}, c\right\rangle}{\left\langle\phi_{m}, \phi_{m}\right\rangle}=c \delta_{m, 0}
$$

Single variables are expanded into a summation of their amplitudes and basis functions,

$$
z_{a}=\sum_{i=0}^{M} \zeta_{a, i} \cos \left(\frac{i \pi x}{\ell}\right)
$$

The normalized inner product can be distributed into this summation, and the orthogonality of basis functions yields each amplitude of that variable appearing in its respective term in the Galerkin expansion with unchanged coefficients,

$$
\frac{\left\langle\phi_{m}, z_{a}\right\rangle}{\left\langle\phi_{m}, \phi_{m}\right\rangle}=\sum_{i=0}^{M} \frac{\zeta_{a, i}}{1+\delta_{m, 0}}\left(\delta_{m, i}+\delta_{m,-i}\right)=\zeta_{a, m}
$$

An arbitrary second-order monomial expands into the following summation by using trigonometric identities to reduce products of cosines into sums of cosines:

$$
\begin{aligned}
z_{a} z_{b} & =\left(\sum_{i=0}^{M} \zeta_{a, i} \cos \left(\frac{i \pi x}{\ell}\right)\right)\left(\sum_{j=0}^{M} \zeta_{b, j} \cos \left(\frac{j \pi x}{\ell}\right)\right) \\
& =\sum_{i=0}^{M} \sum_{j=0}^{M} \zeta_{a, i} \zeta_{b, j} \cos \left(\frac{i \pi x}{\ell}\right) \cos \left(\frac{j \pi x}{\ell}\right) \\
& =\sum_{i=0}^{M} \sum_{j=0}^{M} \frac{\zeta_{a, i} \zeta_{b, j}}{2}\left(\cos \left(\frac{(i+j) \pi x}{\ell}\right)+\cos \left(\frac{(i-j) \pi x}{\ell}\right)\right) .
\end{aligned}
$$

Here the normalized inner product again distributes to each term in the series, yielding

$$
\frac{\left\langle\phi_{m}, z_{a} z_{b}\right\rangle}{\left\langle\phi_{m}, \phi_{m}\right\rangle}=\sum_{i=0}^{M} \sum_{j=0}^{M} \frac{\zeta_{a, i} \zeta_{b, j}}{2\left(1+\delta_{m, 0}\right)}\left(\delta_{m, i+j}+\delta_{m, i-j}+\delta_{m,-i+j}+\delta_{m,-i-j}\right) .
$$


Third-order monomials are expanded in a similar fashion to second-order monomials,

$$
\begin{aligned}
z_{a} z_{b} z_{c} & =\left(\sum_{i=0}^{M} \zeta_{a, i} \cos \left(\frac{i \pi x}{\ell}\right)\right)\left(\sum_{j=0}^{M} \zeta_{b, j} \cos \left(\frac{j \pi x}{\ell}\right)\right)\left(\sum_{k=0}^{M} \zeta_{c, k} \cos \left(\frac{k \pi x}{\ell}\right)\right) \\
& =\sum_{i=0}^{M} \sum_{j=0}^{M} \sum_{k=0}^{M} \zeta_{a, i} \zeta_{b, j} \zeta_{c, k} \cos \left(\frac{i \pi x}{\ell}\right) \cos \left(\frac{j \pi x}{\ell}\right) \cos \left(\frac{k \pi x}{\ell}\right) \\
& =\sum_{i=0}^{M} \sum_{j=0}^{M} \sum_{k=0}^{M} \frac{\zeta_{a, i} \zeta_{b, j} \zeta_{c, k}}{4}\left(\begin{array}{c}
\cos \left(\frac{(i+j+k) \pi x}{\ell}\right)+\cos \left(\frac{(i+j-k) \pi x}{\ell}\right)+ \\
\cos \left(\frac{(i-j+k) \pi x}{\ell}\right)+\cos \left(\frac{(-i+j+k) \pi x}{\ell}\right)
\end{array}\right) .
\end{aligned}
$$

Once more, the normalized inner product distributes to each term in the series, yielding (A.8)

$$
\frac{\left\langle\phi_{m}, z_{a} z_{b} z_{c}\right\rangle}{\left\langle\phi_{m}, \phi_{m}\right\rangle}=\sum_{i=0}^{M} \sum_{j=0}^{M} \sum_{k=0}^{M} \frac{\zeta_{a, i} \zeta_{b, j} \zeta_{c, k}}{4\left(1+\delta_{m, 0}\right)}\left(\begin{array}{c}
\delta_{m, i+j+k}+\delta_{m, i+j-k}+\delta_{m, i-j+k}+\delta_{m,-i+j+k}+ \\
\delta_{m, i-j-k}+\delta_{m,-i+j-k}+\delta_{m,-i-j+k}+\delta_{m,-i-j-k}
\end{array}\right) .
$$

This pattern continues for higher-order polynomials and provides a computationally efficient method of solving and analyzing the Galerkin projection ODEs for the low-order polynomial systems that we find in isothermal Arrhenius kinetics.

Appendix B. Block Jacobian matrix. We evaluate the Jacobian matrix of a Galerkin projection at a spatially homogeneous point. This analysis is applicable for any set of orthogonal basis functions, $\phi_{m}$ for $m=0, \ldots, M$ (as long as $\phi_{0}$ is a spatially homogeneous basis function), and for an arbitrary function, $\dot{\omega}_{i}\left(z_{j}\right)$ for $i, j=1, \ldots, R$. We choose to order the Galerkin projection species amplitude vector first by basis function and then by species by defining $\{i, m\}=m R+i$. Therefore, the vector, $\zeta_{\{i, m\}}=\zeta_{i, m}$ for $i=1, \ldots, R$ and $m=0, \ldots, M$, contains all $R(M+1)$ elements of the species amplitudes sorted in our prescribed fashion. The reaction source terms become ordered as

$$
\dot{\Omega}_{\{i, m\}}=\frac{\left\langle\phi_{m}, \dot{\Omega}_{i}\left(\sum_{n=0}^{M} \zeta_{j, n} \phi_{n}\right)\right\rangle}{\left\langle\phi_{m}, \phi_{m}\right\rangle} \text { for } i, j=1, \ldots, R \text { and } m=0, \ldots, M
$$

To find the Jacobian of a point in the spatially homogeneous subspace, $\zeta_{i, m}^{S H}$ (where $\zeta_{i, m}^{S H}=0$ for $m>0$ ), we then take the partial derivative of the Galerkin projection with respect to an arbitrary species amplitude,

$$
\mathbf{J}_{\{i, m\},\{j, n\}}=\left.\frac{\partial}{\partial \zeta_{j, n}}\left(\frac{\left\langle\phi_{m}, \dot{\Omega}_{i}\left(\sum_{n=0}^{M} \zeta_{j, n} \phi_{n}\right)\right\rangle}{\left\langle\phi_{m}, \phi_{m}\right\rangle}-\frac{m^{2} \pi^{2} D}{\ell^{2}} \zeta_{i, m}\right)\right|_{\zeta=\zeta^{S H}} .
$$

We can distribute the partial derivative into the linear operators and apply them at the point in the spatially homogeneous subspace to get

$$
\mathbf{J}_{\{i, m\},\{j, n\}}=\frac{\left\langle\phi_{m},\left.\frac{\partial}{\partial \zeta_{j, n}} \dot{\Omega}_{i}\left(\sum_{\hat{n}=0}^{M} \zeta_{\hat{j}, \hat{n}} \phi_{\hat{n}}\right)\right|_{\zeta=\zeta^{S H}}\right\rangle}{\left\langle\phi_{m}, \phi_{m}\right\rangle}-\left.\frac{m^{2} \pi^{2} D}{\ell^{2}} \frac{\partial \zeta_{i, m}}{\partial \zeta_{j, n}}\right|_{\zeta=\zeta^{S H}} .
$$

Copyright $\odot$ by SIAM. Unauthorized reproduction of this article is prohibited. 
The partial derivative in the diffusion term can be reduced to a Kronecker delta function,

$$
\mathbf{J}_{\{i, m\},\{j, n\}}=\frac{\left\langle\phi_{m},\left.\frac{\partial}{\partial \zeta_{j, n}} \dot{\Omega}_{i}\left(\sum_{\hat{n}=0}^{M} \zeta_{\hat{j}, \hat{n}} \phi_{\hat{n}}\right)\right|_{\zeta=\zeta^{S H}}\right\rangle}{\left\langle\phi_{m}, \phi_{m}\right\rangle}-\frac{m^{2} \pi^{2} D}{\ell^{2}} \delta_{\{i, m\},\{j, n\}} .
$$

Then we take the derivative of the arbitrary nonlinear reaction function. The derivative can be reduced to be with respect to $z_{j}$ since it is evaluated at the spatially homogeneous $z^{S H}$. Because of the chain rule, the basis function, $\phi_{n}$, becomes a coefficient of the derivative, yielding

$$
\mathbf{J}_{\{i, m\},\{j, n\}}=\frac{\left\langle\phi_{m}, \phi_{n} \frac{\partial \dot{\Omega}_{i}}{\partial z_{j}}\right\rangle}{\left\langle\phi_{m}, \phi_{m}\right\rangle}-\frac{m^{2} \pi^{2} D}{\ell^{2}} \delta_{\{i, m\},\{j, n\}} .
$$

Finally, the orthogonality of the basis functions will yield another Kronecker delta function, resulting in

$$
\mathbf{J}_{\{i, m\},\{j, n\}}=\frac{\partial \dot{\Omega}_{i}}{\partial z_{j}} \delta_{m, n}-\frac{m^{2} \pi^{2} D}{\ell^{2}} \delta_{\{i, m\},\{j, n\}}
$$

This Jacobian is in the form of a block diagonal matrix. The first term, $\left(\partial \dot{\Omega}_{i} / \partial z_{j}\right) \delta_{m, n}$, populates the $R \times R$ diagonal blocks with the spatially homogeneous Jacobian matrix. The second term, $\left(m^{2} \pi^{2} D / \ell^{2}\right) \delta_{\{i, m\},\{j, n\}}$, yields a diffusion component along the diagonal of each block.

Appendix C. Poincaré sphere. To evaluate the dynamics at infinity, our domain is projected onto the Poincaré sphere [48], mapping $\zeta \in \mathbb{R}^{M+1} \mapsto \boldsymbol{\eta} \in \mathbb{R}^{M+2}$ via the coordinate transformation,

$$
\begin{aligned}
\eta_{i} & =\frac{\zeta_{i}}{\sqrt{1+\sum_{m=0}^{M} \zeta_{m}^{2}}} \text { for } i=0, \ldots, M, \\
\eta_{M+1} & =\frac{1}{\sqrt{1+\sum_{m=0}^{M} \zeta_{m}^{2}}} .
\end{aligned}
$$

Under this transformation, the $\eta$-coordinates are constrained by

$$
\sum_{i=0}^{M+1} \eta_{i}^{2}=1
$$

which means that the $\zeta$-coordinate system is mapped onto an $(M+1)$-dimensional unit hypersphere in $\eta$-space. The inverse transformation exists,

$$
\zeta_{i}=\frac{\eta_{i}}{\eta_{M+1}} \text { for } i=0, \ldots, M .
$$

When we examine only $\eta_{i}$ for $i=0, \ldots, M$, we project the unit hypersphere into an $(M+1)$ dimensional space and the constraint in (C.2) becomes an inequality,

$$
\sum_{i=0}^{M} \eta_{i}^{2} \leq 1
$$

Copyright (c) by SIAM. Unauthorized reproduction of this article is prohibited. 
For this transformation, in the limit as $\zeta_{i} \rightarrow \infty$, for any $i=0, \ldots, M, \eta_{i}$ becomes 1 . More generally, in the limit as any set of $\zeta_{i} \rightarrow \infty$, the resulting transformed variables, $\eta_{i}$, are finite and constrained by

$$
\sum_{i=0}^{M} \eta_{i}^{2}=1
$$

Therefore, the points at infinity in $\zeta$-coordinates are mapped onto an $M$-dimensional unit hypersphere in the $\eta$-coordinates. For finite values of $\zeta_{i}$,

$$
\sum_{i=0}^{M} \eta_{i}^{2}<1
$$

therefore, all of the finite dynamics in the $\zeta$-coordinates are contained within the $M$-dimensional unit sphere in the $\eta$-coordinates. In the Poincaré sphere coordinate transformation, the entire $\zeta$-coordinate system, both finite and infinite, is mapped onto a unit sphere. This allows the entire system's dynamics to be examined, including the dynamics at infinity.

\section{REFERENCES}

[1] J. M. Powers, Review of multiscale modeling of detonation, J. Propul. Power, 22 (2006), pp. 1217-1229.

[2] J. F. GRIfFiths, Reduced kinetic-models and their application to practical combustion systems, Prog. Energy Combust. Sci., 21 (1995), pp. 25-107.

[3] T. LU AND C. K. LAW, Toward accommodating realistic fuel chemistry in large-scale computations, Prog. Energy Combust. Sci., 35 (2009), pp. 192-215.

[4] U. MaAs And S. B. Pope, Simplifying chemical kinetics: Intrinsic low-dimensional manifolds in composition space, Combust. Flame, 88 (1992), pp. 239-264.

[5] S. H. LAM, The CSP method for simplifying kinetics, Int. J. Chem. Kinet., 26 (1994), pp. 461-486.

[6] V. Bykov, V. Gol'Dshtein, And U. MaAs, Simple global reduction technique based on decomposition approach, Combust. Theory Model., 12 (2008), pp. 389-405.

[7] A. N. Gorban And I. V. Karlin, Method of invariant manifold for chemical kinetics, Chem. Eng. Sci., 58 (2003), pp. 4751-4768.

[8] D. LeBIEdZ, Computing minimal entropy production trajectories: An approach to model reduction in chemical kinetics, J. Chem. Phys., 120 (2004), pp. 6890-6897.

[9] Z. Ren, S. B. Pope, A. Vladimirsky, And J. M. Guckenheimer, The invariant constrained equilibrium edge preimage curve method for the dimension reduction of chemical kinetics, J. Chem. Phys., 124 (2006), 114111.

[10] S. J. FrASER, The steady state and equilibrium approximations: A geometrical picture, J. Chem. Phys., 88 (1988), pp. 4732-4738.

[11] M. R. Roussel And S. J. Fraser, Geometry of the steady-state approximation: Perturbation and accelerated convergence methods, J. Chem. Phys., 93 (1990), pp. 1072-1081.

[12] M. R. Roussel and S. J. Fraser, On the geometry of transient relaxation, J. Chem. Phys., 94 (1991), pp. $7106-7113$.

[13] M. J. Davis And R. T. Skodje, Geometric investigation of low-dimensional manifolds in systems approaching equilibrium, J. Chem. Phys., 111 (1999), pp. 859-874.

[14] H. G. KAPER AND T. J. KAPER, Asymptotic analysis of two reduction methods for systems of chemical reactions, Phys. D, 165 (2002), pp. 66-93.

[15] C. W. Gear, T. J. Kaper, I. G. Kevrekidis, and A. Zagaris, Projecting to a slow manifold: Singularly perturbed systems and legacy codes, SIAM J. Appl. Dyn. Syst., 4 (2005), pp. 711-732.

Copyright $\odot$ by SIAM. Unauthorized reproduction of this article is prohibited. 
[16] F. Creta, A. Adrover, S. Cerbelli, M. Valorani, and M. Giona, Slow manifold structure in explosive kinetics. 1. Bifurcations of points-at-infinity in prototypical models, J. Phys. Chem. A, 110 (2006), pp. 13447-13462.

[17] M. Giona, A. Adrover, F. Creta, and M. Valorani, Slow manifold structure in explosive kinetics. 2. Extension to higher dimensional systems, J. Phys. Chem. A, 110 (2006), pp. 13463-13474.

[18] A. N. Al-Khateeb, J. M. Powers, S. Paolucci, A. J. Sommese, J. A. Diller, J. D. Hauenstein, And J. D. Mengers, One-dimensional slow invariant manifolds for spatially homogenous reactive systems, J. Chem. Phys., 131 (2009), 024118.

[19] D. Lebiedz, J. Siehr, And J. Unger, A variational principle for computing slow invariant manifolds in dissipative dynamical systems, SIAM J. Sci. Comput., 33 (2011), pp. 703-720.

[20] U. MAAS AND S. B. Pope, Implementation of simplified chemical kinetics based on intrinsic lowdimensional manifolds, P. Combust. Inst., 24 (1992), pp. 103-112.

[21] U. MAas And S. B. Pope, Laminar flame calculations using simplified chemical kinetics based on intrinsic low-dimensional manifolds, P. Combust. Inst., 25 (1994), pp. 1349-1356.

[22] Z. Ren, S. B. Pope, A. Vladimirsky, and J. M. Guckenheimer, Application of the ICE-PIC method for the dimension reduction of chemical kinetics coupled with transport, P. Combust. Inst., 31 (2007), pp. 473-481.

[23] Z. REn AND S. B. Pope, Transport-chemistry coupling in the reduced description of reactive flows, Combust. Theory Model., 11 (2007), pp. 715-739.

[24] S. H. LAM, Reduced chemistry-diffusion coupling, Combust. Sci. Technol., 179 (2007), pp. 767-786.

[25] M. Hadjinicolaou And D. A. Goussis, Asymptotic solution of stiff PDEs with the CSP method: The reaction diffusion equation, SIAM J. Sci. Comput., 20 (1999), pp. 781-810.

[26] S. Singh, J. M. Powers, And S. PaOlucci, On slow manifolds of chemically reactive systems, J. Chem. Phys., 117 (2002), pp. 1482-1496.

[27] V. Bykov and U. MAas, The extension of the ILDM concept to reaction-diffusion manifolds, Combust. Theory Model., 11 (2007), pp. 839-862.

[28] M. J. DAvis, Low-dimensional manifolds in reaction-diffusion equations. 1. Fundamental aspects, J. Phys. Chem. A, 110 (2006), pp. 5235-5256.

[29] M. J. DAVIS, Low-dimensional manifolds in reaction-diffusion equations. 2. Numerical analysis and method development, J. Phys. Chem. A, 110 (2006), pp. 5257-5272.

[30] A. Adrover, F. Creta, M. Giona, and M. Valorani, Stretching-based diagnostics and reduction of chemical kinetic models with diffusion, J. Comput. Phys., 225 (2007), pp. 1442-1471.

[31] J. C. Robinson, Infinite-Dimensional Dynamical Systems, Cambridge University Press, Cambridge, UK, 2001.

[32] R. Temam, Infinite-Dimensional Dynamical Systems in Mechanics and Physics, Springer-Verlag, Berlin, 1997.

[33] E. N. Lorenz, Deterministic nonperiodic flow, J. Atmospheric Sci., 20 (1963), pp. 130-141.

[34] D. Henry, Geometric Theory of Semilinear Parabolic Problems, Springer-Verlag, Berlin, 1981.

[35] G. Fusco AND J. K. HALE, Slow-motion manifolds, dormant instability, and singular perturbations, J. Dynam. Differential Equations, 1 (1989), pp. 75-94.

[36] L. V. Kalachev, H. G. Kaper, T. J. Kaper, N. Popovic, and A. Zagaris, Reduction for MichaelisMenten-Henri kinetics in the presence of diffusion, in Proceedings of the International Conference in Honor of Jacqueline Fleckinger, Toulouse, France, 2006, Electron. J. Differ. Equ. Conf. 16, 2007, pp. $155-184$.

[37] A. N. Yannacopoulos, A. S. Tomlin, J. Brindley, J. H. Merkin, and M. J. Pilling, The use of algebraic sets in the approximation of inertial manifolds and lumping in chemical kinetic systems, Phys. D, 84 (1995), pp. 421-449.

[38] T. I. Seidman And L. V. Kalachev, A one-dimensional reaction/diffusion system with a fast reaction, J. Math. Anal. Appl., 209 (1997), pp. 392-414.

[39] L. V. KAlacheV AND T. I. SEIDMAN, Singular perturbation analysis of a stationary diffusion/ reaction system whose solution exhibits a corner-type behavior in the interior of the domain, J. Math. Anal. Appl., 288 (2003), pp. 722-743.

[40] T. I. SEIDMAn, Interface conditions for a singular reaction-diffusion system, Discrete Contin. Dyn. Syst. Ser. S, 2 (2009), pp. 631-643.

Copyright (C) by SIAM. Unauthorized reproduction of this article is prohibited. 
[41] B. SporTisse, An analysis of operator splitting techniques in the stiff case, J. Comput. Phys., 161 (2000), pp. $140-168$.

[42] W. Hundsdorfer and J. G. Verwer, Numerical Solutions of Time-Dependent Advection-DiffusionReaction Equations, Springer-Verlag, Berlin, 2003.

[43] D. L. Ropp, J. N. Shadid, And C. C. OBer, Studies of the accuracy of time integration methods for reaction-diffusion equations, J. Comput. Phys., 194 (2004), pp. 544-574.

[44] A. N. Al-Khateeb, J. M. Powers, And S. Paolucci, Analysis of the spatio-temporal scales of laminar premixed flames near equilibrium, Combust. Theory Model., 17 (2013), pp. 76-108.

[45] R. B. Bird, W. E. Stewart, And E. N. Lightfoot, Transport Phenomena, John Wiley \& Sons, New York, 1960.

[46] B. A. Finlayson, The Method of Weighted Residuals and Variational Principles, with Application in Fluid Mechanics, Heat and Mass Transfer, Academic Press, New York, 1972.

[47] S. H. LAm ANd J. Bellan, On de-coupling of Shvab-Zel'dovich variables in the presence of diffusion, Combust. Flame, 132 (2003), pp. 691-696.

[48] L. Perko, Differential Equations and Dynamical Systems, Springer-Verlag, Berlin, 2001.

[49] R. J. Kee, F. M. Rupley, And J. A. Miller, The CHEMKIN Thermodynamic Database, Tech. Report SAND87-8215B, Sandia National Laboratories, Livermore, CA, 1992.

[50] R. J. Kee, L. G. Dixon, J. Warnatz, M. E. Coltrin, And J. A. Miller, A Fortran Computer Code Package for the Evaluation of Gas-Phase Multicomponent Transport Properties, Tech. Report SAND86-8246, Sandia National Laboratories, Livermore, CA, 1991.

Copyright $\odot$ by SIAM. Unauthorized reproduction of this article is prohibited. 